$N^{\circ} 291 \quad$ ISSN 0104-8910

INTEGRAÇÄO, CRESCIMENTO E BEM-ESTAR

Marcelo Leite de Moura e Silva

Pedro C. Ferreira

Outubro de 1996 


\title{
Integração, Crescimento e Bem-estar
}

\author{
Marcelo Leite de Moura e Silva \\ Pontificia Universidade Católica de Minas Gerais \\ \& University of Chicago
}

\author{
Pedro C. Ferreira \\ Escola de Pós-Graduação em Economia \\ Fundação Getúlio Vargas / RJ
}

\begin{abstract}
Resumo: Neste artigo desenvolve-se um modelo de crescimento econômico endógeno para o caso de duas economias integradas levando-se em conta a presença de custos de transporte na forma de iceberg costs. A presença de tais custos juntamente com a existência de ganhos de escala terá um efeito negativo sobre a taxa de crescimento dos paises integrados e exercerá um importante efeito sobre a localização da produção de insumos intermediários. Demonstra-se, no entanto, que um processo de integração com custos de transporte ainda leva a um aumento da taxa de crescimento de estado estacionário devido ao aumento do mercado consumidor de insumos. $O$ ganho de bem-estar decorrente da integração dependerá das dotaçoes de cada país, na medida em que o nível de consumo per capita após a integração depende destas dotacoes. Adicionalmente, apresenta-se uma simulaça de ganhos em termos de taxa de crescimento do PNB e de bem-estar para uma integração econômica entre Brasil e Argentina.
\end{abstract}

\footnotetext{
- Agradecimentos: Os autores agradecem os comentários do professor Renato Fragelli e especialmente dos professores Afonso Arinos de Mello Franco Neto e Rodolfo Manuelli, também agradecem ao CNPq pelo apoio financeiro.
} 


\section{I - Introdução}

David Ricardo no início do século XIX já mencionava a existência de vantagens comparativas como um motivo para que o livre comércio gerasse um ganho em termos de eficiência alocativa aos paises. No entanto, não é apenas uma maior eficiência alocativa que parece motivar o livre comércio, ganhos em termos de extensão de mercado, exploração de ganhos de escala, aumento da competitividade e um maior incentivo a busca do progresso tecnológico seriam também outras conseqüências do livre comércio. Estes são fatores, por sua vez, geradores do crescimento econômico em alguns recentes modelos de crescimento endógeno como em Barro (1995), Romer (1990), Grossman \& Helpman (1990) e Aghion \& Howitt (1992). Desta forma a integração econômica através de livre comércio de bens e livre mobilidade de fatores de produção se mostra como impulsionadora do crescimento econômico dos paises que se integram.

Associações de livre comércio tem aparecido cada vez mais no cenário mundial que se manifesta através da formação de blocos de livre comércio como o EC, NAFTA, Apec e Mercosul $^{1}$. O presente artigo pretende enfatizar três efeitos econômicos do processo de integração regional. Primeiro, com a integração econômica haverá uma extensão automática do mercado, o que propiciará maiores lucros e incentivará os investimentos em pesquisa e desenvolvimento (P\&D). Isto é, com a integração econômica a taxa de retorno da atividade de P\&D irá subir, dado que os insumos inventados podem ser vendidos tanto para o mercado doméstico quanto para o mercado externo sem que incida sobre eles nenhum imposto sobre sua comercialização. Maior investimento em P\&D, por sua vez, implicará em maior progresso tecnológico, o que aumentará a taxa de crescimento econômico dos países integrados.

Em segundo lugar, a presença de custos de transporte sobre o comércio internacional juntamente com ganhos de escala na produção de insumos leva a uma concentração da produção destes no país com maior mercado. Por outro lado, um segundo efeito será do custo de transporte será o de diminuir o aumento do mercado consumidor e portanto a recompensa da atividade de P\&D. Como em livre comércio as aliquotas de importação serão zero, o único custo relevante para se comercializar uma mercadoria será o de transporte. Deste forma, como 
o transporte de mercadorias atua consumindo fatores produtivos e diminuindo a produtividade marginal destes, haverá um efeito negativo sobre a taxa de crescimento da economia e do emprego de fatores de produção.

Finalmente, a abertura comercial irá promover o acesso a produtos e tecnologias que não eram disponíveis no mercado interno. Isto levará a um aumento da produtividade do trabalho e do nível de produção através da utilização destes insumos importados.

Para explicar tais fenômenos iremos utilizar um modelo de crescimento endógeno na tradição dos modelos de Romer (1990) onde o tamanho do mercado e a quantidade de insumos distintos possuem um papel fundamental na determinação do nivel e da taxa de crescimento do produto. Um aspecto importante do modelo é que este enfatiza a idéia defendida por Schumpeter de que a firma ao criar ou inovar determinado produto passa a deter direitos de monopólio sobre tal produto. Desta forma o fluxo de lucros gerado pelo monopólio é que irá motivar as firmas a lançar novos produtos e isto será o motor do progresso tecnológico.

$O$ aumento do mercado externo provoca um aumento do investimento em P\&D que por sua vez leva a um aumento da taxa de crescimento do produto e do consumo dos países integrados. No entanto, isto não irá representar necessariamente um ganho de bem-estar na medida em que uma maior taxa de crescimento requer um maior nivel de investimento e consequentemente uma maior abstenção de consumo presente. Por outro lado o nível de consumo poderá aumentar devido ao aumento do nível de produção gerado pela adição de insumos importados ao conjunto de insumos domésticos. Qual efeito será dominante, e se haverá um ganho de bem-estar com a integração, dependerá da quantidade de capital humano e nível tecnológico de um país em relação ao outro, bem como do custo de transporte e da taxa de desconto intertemporal do individuo. Será necessário portanto analisar o efeito da integração sobre o nível - que pode cair - e a taxa de crescimento - que sempre aumenta - do consumo para saber se o ganho líquido de bem-estar é positivo..

O modelo, entretanto, não possui uma forma fechada onde se possa concluir qual o efeito dominante sobre o bem-estar e também que se possa ver sem ambigüidade qual o efeito de variações de parâmetros externos sobre crescimento. Desta forma, a partir dos resultados do modelo iremos simular, utilizando estimativas dos parâmetros do modelo, efeitos de integração

'EC, European Comunity, NAFTA, North American Free Trade Association; Apec, Asia Pacific Economic 
entre Brasil e Argentina. O objetivo desta parte do trabalho é mais ilustrativo do que preditivo visando predominantemente obter resultados qualitativos para a variação de bem-estar e de taxas de crescimento a partir das hipóteses do modelo.

O trabalho se divide em cinco seções, incluindo-se esta introdução. Na próxima seção será apresentado o modelo de integração; na terceira seção será analisado o efeito da integração e de variações dos parâmetros do modelo sobre o bem-estar; na quarta seção será apresentada a simulação dos resultados do modelo para o caso de integração entre Brasil e Argentina. Por fim, na última seção, serão apresentadas as conclusões finais do trabalho.

\section{I - Aumento na diversidade de insumos, integração.}

Utilizando a estrutura básica do modelo para o caso de um país isolado desenvolvido por Barro \& Sala-i-Martin (1995) iremos desenvolver um modelo de crescimento para duas economias integradas ${ }^{2}$. O processo de integração aqui analisado será definido como o estabelecimento de livre comércio entre os países integrados sem a cobrança de nenhum tipo de imposto, supondo livre movimentação de capitais mas sem migração da mão-de-obra.

Para incorporar os efeitos da integração a função de produção de cada firma será expandida de forma a incorporar não apenas os insumos domésticos como também os insumos importados. $\mathrm{O}$ único custo sobre o comércio de insumos entre os países será o de transporte e sua inclusão será feita através da suposição de que tal custo é da forma de "iceberg cost." Isto significa que quando o importador compra uma unidade do bem intermediário produzido em outro país ele receberá $\phi$ unidades do bem importado, onde $\phi$ é um número real entre zero e um. $O$ custo de transporte é portanto 1- $\phi$ unidades do bem importado. Adicionalmente supomos que o serviço de transporte é realizado por um terceiro país de forma que não gera receita para os países em integração.

A função de produção para a firma i do país 1 será dada por:

Cooperation agreement, Mercosul, Mercado Comum do Cone Sul.

${ }^{2}$ A generalização para o caso de n economias integradas é imediata, basta considerar a economia 2 como sendo o conjunto de n-1 paises e a economia 1 como um país isolado, para verificar o efeito da integraça em cada um dos n paises basta alternar cada pais como sendo a economia 1. 


$$
Y_{1 i}=A L_{1 i}^{1-\alpha}\left[\int_{0}^{N(1)}\left(X_{1 i j}\right)^{\alpha} d j+\int_{0}^{N(2)}\left(M_{1 i j}\right)^{\alpha} d j\right]
$$

onde $Y_{1 i}$ é o produto final da firma $i$ instalada no pás $1 ; L_{1 i}$ a mão-de-obra empregada pela firma $\mathrm{i}$ instalada no pais $1 ; \mathrm{N}_{1}$ a quantidade de insumos diversos existentes no país 1 (ou nivel tecnológico do país 1); $\mathrm{N}_{2}$ a quantidade de insumos diversos existentes no país $2^{3}$ (ou nivel tecnológico do país 2); $X_{\mathrm{lij}}$ a quantidade do insumo $\mathrm{j}$, produzido por uma firma de propriedade do país 1, utilizado pela firma i e $\mathrm{M}_{\mathrm{ij}}$ a quantidade do insumo $\mathrm{j}$, produzido por uma firma de propriedade do país 2 , utilizado pela firma i. Será assumido que o estoque de mão-de-obra é fixo e sua oferta é inelástica em ambos países.

Analogamente temos que: $\mathrm{X}_{2 \mathrm{ij}}$ é a quantidade do insumo $\mathrm{j}$, produzido por uma firma de propriedade do país 2, utilizado pela firma $\mathrm{i}$ instalada no país $2 \mathrm{e} \mathbf{M}_{2 \mathrm{ij}}$ a quantidade do insumo $\mathrm{j}$, produzido por uma firma de propriedade do pais 1 , utilizado pela firma i instalada no pais 2 .

$O$ produtor do bem final contratará mão-de-obra e irá adquirir insumos produzidos internamente $\mathrm{e}$ insumos importados para produzir. Ele opera em concorrência perfeita, tomando o preço dos fatores de produção como dados e da solução de seu problema obtém-se a demanda por bens intermediários produzidos internamente e importados. $O$ problema do produtor de bem final é portanto:

$$
\operatorname{Max} \quad Y_{1 i}-w_{1} L_{1 i}-\int_{0}^{N(1)}\left(P_{1 j} X_{1 i j}\right) d j-\int_{0}^{N(2)}\left(P_{2 j} M_{1 i j}\right) d j
$$

A condição de primeira ordem para a maximização do lucro do produtor do bem de consumo final localizado no país 1 leva a:

1) Demanda pelo bem doméstico,

$$
\begin{aligned}
& P_{1 j}=\operatorname{Pmg}\left(X_{1 i j}\right) \Rightarrow X_{1 i j}=L_{1 i}\left(A \alpha / P_{1 j}\right)^{1 /(1-\alpha)} \\
& X_{1 j}=\sum_{i} X_{1 i j}=\left(A \alpha / P_{1 j}\right)^{1 /(1-\alpha)} \sum_{i j} L_{1 i}=\left(A \alpha / P_{1 j}\right)^{1 /(1-\alpha)} L_{1}
\end{aligned}
$$

\footnotetext{
${ }^{3}$ Para efeito de simplificaçăo iremos considerar não redundância entre a diversidade de insumos dos dois países, ou seja, os insumos intermediários existentes no país 1 são totalmente distintos dos insumos existentes no país 2. Esta hipótese não é irrealista se consideramos que as funçðes de produção apresentadas nesta seção representam a parte da economia de cada país que possui vantagens comparativas em relacão ao outro pais.
} 
2) No caso da demanda pelo bem importado temos que levar em conta o efeito do custo de transporte. Como o importador recebe $\phi$ unidades por cada unidade comprada a produtividade marginal de cada unidade de insumo importado será multiplicada por $\phi$. Logo, a demanda do país 1 por bens intermediários produzidos no país 2 será:

$$
\begin{aligned}
& P_{2 j}=\phi P M g\left(M_{1 i j}\right) \Rightarrow M_{1 i j}=L_{1 i}\left(A \alpha \phi / P_{2 j}\right)^{1 /(1-\alpha)} \\
& M_{1 j}=\Sigma_{i} M_{1 i j}=\left(A \alpha / P_{2 j}\right)^{1 /(1-\alpha)} \sum_{i} L_{1 i}=\left(A \alpha / P_{2 j}\right)^{1 /(1-\alpha)} \phi^{1 /(1-\alpha)} L_{1}
\end{aligned}
$$

A demanda do bloco pelo insumo j que é produzido no país 1 será dada por $\aleph_{\mathrm{ij}}=\mathrm{X}_{1 \mathrm{j}}+\mathrm{M}_{2 \mathrm{j}}$. Utilizando a equação (1.3) e reformulando a equação (1.4) para $\mathbf{M}_{2 \mathrm{j}}$ - que representa a demanda do país 2 pelo insumo j produzido no país 1 - obtemos a curva de demanda mundial pelo bem $\mathbf{j}$.

$$
\begin{aligned}
& \aleph_{1 j}=\left(A \alpha / P_{1 j}\right)^{1 /(1-\alpha)} L^{W} \\
& L^{W}=L_{1}+\phi^{1 /(1-\alpha)} L_{2}
\end{aligned}
$$

Das equações (1.5) e (1.6) concluímos que o aumento do custo de transporte (queda de ф) implica em uma diminuição da demanda externa pelo insumo j, já que,

$$
\left(\partial \aleph_{1 j} / \partial \phi\right)=[1 /(1-\alpha)]\left(A \alpha / P_{1 j}\right)^{1 /(1-\alpha)} \phi^{\alpha /(1-\alpha)} L_{2}>0
$$

A intuição por trás deste resultado é que o aumento do custo de transporte implica em uma queda da produtividade marginal do insumo importado, e dado que o preço do insumo está fixo, como será visto adiante, a demanda externa pelo insumo irá cair.

O custo de se inventar um novo insumo e aumentar o nivel tecnológico no país 1 ou no país 2 é fixo e dado por $\boldsymbol{\eta}$ e o custo para produzir o insumo já inventado para comercialização é de uma unidade de bem de consumo para cada unidade de insumo. Além disso existe um custo fixo de produção que é função do número de firmas utilizadas para produzir tal insumo. Desta forma, o lucro em valor presente do monopolista que já incorreu no custo fixo de inventar um novo insumo é dado por: 


$$
V_{1}(t)=\int_{0}^{+\infty}\left[\left(P_{1 j}-1\right) N_{1 j} e^{-R 1(v, t)(v-t)}-\theta(n-1)\right] d v
$$

onde: $R 1(v, t)=\int_{t}^{v} r(w) d w /(v-t)$ ( $r$ é a taxa de juros), $n$ é o número de firmas utilizadas para produzir o insumo ( $n$ é um número inteiro positivo) e $\theta$ é um parâmetro de custo tal que:

$$
\theta>\left[\left(1-\phi^{1 /(1-\alpha)}\right) / \eta\right]\left(A \alpha^{2}\right)^{1 /(1-\alpha)}\left(\alpha^{-1}-1\right) \min \left\{L_{1}, L_{2}\right\}>0
$$

A escolha do número de firmas será $\mathrm{n}=1$. A única vantagem de se aumentar o número de firmas seria a de evitar o custo de transporte ao instalar uma firma em cada país. No entanto, a condição (1.7) reflete a existência de um ganho de escala decorrente da utilização de apenas uma firma, o que torna não lucrativa a instalação de uma segunda firma para evitar o custo de transporte.

O monopolista do país 1 irá escolher $P_{1 j}$ de forma a maximizar seu lucro. Dadas as equações (1.5) e (1.7) o problema torna-se,

$$
\operatorname{Max}\left(P_{1 j}-1\right)\left(A \alpha / P_{1 j}\right)^{1 /(1-\alpha)} L^{w}
$$

Uma vez que o preço de maximização não irá depender do tamanho do mercado consumidor, a solução do problema dado por (1.8) leva ao preço de equilíbrio

$$
\mathbf{P}_{1 \mathrm{j}}=\mathbf{P}=1 / \alpha
$$

O preço de monopólio, como no modelo para economias fechadas, é um preço de mark-up de $1 / \alpha$ sobre o custo marginal, que é de uma unidade de bem de consumo final.

Aplicando (1.5) e (1.9) na equação (1.7) obtemos:

$$
V_{1}(t)=\left(A \alpha^{2}\right)^{1 /(1-\alpha)} L^{w}\left(\alpha^{-1}-1\right) \int_{0}^{+\infty} e^{-R 1(v, t)(v-t)} d v
$$


A hipótese de que os produtores de bens intermediários operam em concorrência monopolística implica que o valor presente dos lucros de monopólio irá se igualar ao custo de se entrar no negócio (que é igual ao custo de inventar o novo insumo) dado por $\eta$ :

$$
\eta=\left(A \alpha^{2}\right)^{1 /(1-\alpha)} L^{w}\left(\alpha^{-1}-1\right) \int_{0}^{+\infty} e^{-R 1(v, t)(v-1)} d v
$$

Como a expressão a direita se iguala a uma constante e todos seus termos fora da integral são também constantes, concluimos que a taxa de retorno é uma constante dada por:

$$
r_{1}{ }^{\text {int }}=\left[\left(L_{1}+\phi^{1 /(1-\alpha)} L_{2}\right) / \eta\right]\left(A \alpha^{2}\right)^{1 /(1-\alpha)}\left(\alpha^{-1}-1\right)
$$

Note que a taxa de retorno acima corresponde a taxa de retorno de uma firma produtora de bens intermediários localizada no país 1 . Consequentemente a taxa de retorno das firmas de bens intermediários localizadas no país 2 será de:

$$
r_{2}{ }^{\text {int. }}=\left[\left(L_{2}+\phi^{1 /(1-\alpha)} L_{1}\right) / \eta\right]\left(A \alpha^{2}\right)^{1 /(1-\alpha)}\left(\alpha^{-1}-1\right)
$$

Devido a suposição de escala na produção de insumos cada firma produz um único insumo e duas firmas não produzem o mesmo insumo. Além disso, dado que os dois países estão integrados uma firma de propriedade de um residente de um determinado pais pode se instalar em qualquer um dos dois países remetendo livremente os lucros para o país do seu proprietário. A integração, no entanto, implica em livre movimentação de capitais. Portanto, por não arbitragem a taxa de juros das economias integradas deverá ser a mesma. Para encontrarmos a taxa de juros de equilibrio do modelo vamos inicialmente considerar como $\pi_{1}$ e $\pi_{2}$ o fluxo de lucro em valor presente de uma firma produtora de bens intermediários localizada no país 1 e no país 2 respectivamente, ou seja,

$$
\begin{aligned}
& \pi_{i}(r)=\left(A \alpha^{2}\right)^{1 /(1-\alpha)}\left(L_{i}+\phi^{1 /(1-\alpha)} L_{i^{*}}\right)\left(\alpha^{-1}-1\right) \int_{0}^{+\infty} e^{-t} d v-\eta, \quad i=1,2 \text {. i*=1 se } i=2 \text { e } \\
& i^{*}=2 \text { se } i=1 .
\end{aligned}
$$


O lucro em valor presente de uma determinada firma será portanto função da taxa de juros do bloco, que é dada por $r$. Para valores de $r^{4}$ tais que $r<r_{2}{ }^{\text {int }}, \pi_{i}(r)>0(i=1,2)$, de forma que tais valores de $\mathrm{r}$ não suportam um equilíbrio de concorrência monopolística com livre entrada. Para $r=r_{2}{ }^{\text {int }}, \pi_{1}(r)>0$ e $\pi_{2}(r)=0$, o que também não pode ser um valor de equilíbrio para $r$. No caso de $r_{2}{ }^{\text {int }}<r<r_{1}{ }^{\text {int }}$ teremos $\pi_{1}(r)>0$ e $\pi_{2}(r)<0$, e para $r>r_{1}{ }^{\text {int }}$ temos $\pi_{1}(r)<0$ e $\pi_{2}(r)<0$, e ambos casos não podem ser solução de equilibrio. Finalmente para $r=r_{1}^{\text {int }}$ vale que $\pi_{1}(r)=0$ e $\pi_{2}(r)<0$. Portanto, o único valor que pode sustentar um equilíbrio em concorrência monopolística e um investimento positivo na produção de insumos intermediários é o da taxa de juros internacional dada por $r=r_{1}{ }^{\text {int }}$, ou seja,

$$
r=\left[\left(L_{1}+\phi^{1 /(1-\alpha)} L_{2}\right) / \eta\right]\left(A \alpha^{2}\right)^{1 /(1-\alpha)}\left(\alpha^{-1}-1\right)
$$

Este resultado nos diz que o país com maior estoque de capital humano terá o maior mercado consumidor e atrairá a totalidade das indústrias produtoras de bens intermediários 5 . Devido ao fato de termos $\pi_{1}(r)=0$ e $\pi_{2}(r)<0$ não se investirá no país 2 e a produção irá se concentrar no país 1 . Tal resultado justifica o fato de que indústrias que possuam ganhos de escala no tamanho de planta, na ausência de outros incentivos (como os fiscais), venham a se localizar próximo dos mercados consumidores para arcar com menores custos de transporte.

O fluxo descontado dos lucros do monopolista irá se igualar ao custo de invenção do novo insumo, $\eta$. Logo, como existem $N_{1}$ unidades produtoras de posse de residentes do país 1 , o valor de mercado das unidades produtoras de propriedade de residentes do país 1 dado por $\eta N_{1}$ e das firmas de residentes do país 2 dado por $\eta N_{2}$.

Para analisar o problema do consumidor representativo é necessário levar em consideração a restrição de recursos de cada um dos países. Para isto é necessário o cálculo do Produto Nacional Bruto de cada pás. Das contas nacionais adaptadas a uma economia sem governo vem que:

\footnotetext{
${ }^{4}$ Suponha, sem perda de generalidade que $L_{1}>L_{2}$. Consequentemente $r_{1}{ }^{\text {int }}>r_{2}{ }^{\text {int }}$.

5 Poderiamos supor irreversibilidade dos investimentos, i.e., o capital já instalado"das firmas não se move, somente os novos negócios surgidos após a integraçăo é que irão, em sua totalidade, se instalar no pais 1 . Desta maneira os resultados do modelo seriam resultados assintóticos.
} 
Valor bruto da produção - Valor das transações intermediárias - Renda líquida enviada ao exterior $=$ Produto Nacional Bruto

O país 1 concentrará todo o gasto com transações intermediárias que representa, neste caso, todos os gastos com insumos para a produção das indústrias de bens finais. Além disso, 0 país 1 terá um renda líquida enviada ao exterior positiva, tal conta irá representar o fluxo de lucro que as empresas de bens intermediários de propriedade de residentes do país 2 e instaladas no país 1 irão enviar ao país 2. Portanto, para o país 1 temos que:

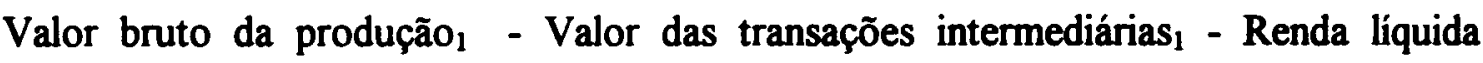
enviada ao exterior ${ }_{1}=\mathrm{PNB}_{1}$, ou seja,

$$
\begin{aligned}
& \quad Y_{1}-\left[N_{1} X_{1}+N_{1} M_{2}+N_{2} X_{2}+N_{2} M_{1}\right]-\left[(P-1)\left(N_{2} X_{2}+N_{2} M_{1}\right)\right]=Y_{1}-N_{1} X_{1}- \\
& N_{1} M_{2}-P_{2} X_{2}-P_{2} M_{1}, \operatorname{logo}: \\
& \quad P N B_{1}=Y_{1}-N_{1} X_{1}-N_{1} M_{2}-P_{2} X_{2}-P_{2} M_{1}
\end{aligned}
$$

Outra maneira de se calcular o PNB é olhando para o lado do dispêndio agregado da economia. No caso da economia sem governo, como neste modelo, o PNB pode ser descrito como:

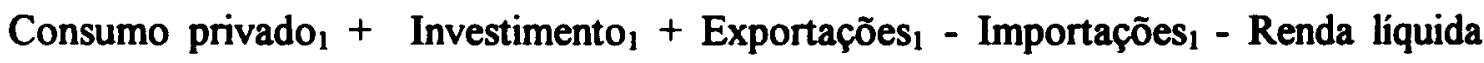

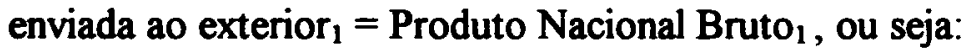

$$
\mathrm{PNB}_{1}=\mathrm{C}_{1}+\eta\left(\mathrm{dN} \mathrm{N}_{1} / \mathrm{dt}\right)+\mathrm{PN}_{1} \mathrm{M}_{2}+\mathrm{N}_{2} \mathrm{X}_{2}-(\mathrm{P}-1) \mathrm{N}_{2} \mathrm{M}_{1}
$$

Por último, o PNB também é obtido pela óptica da renda nacional que nesta economia simplificada é dada por:

Remuneração dos assalariados + Rendas provenientes de propriedade $=$ Produto Nacional Bruto, fazendo o cálculo para o país 1, tenho:

$$
\mathrm{PNB}_{1}=\mathrm{w}_{1} \mathrm{~L}_{1}+\mathrm{mN}_{1}
$$

Fazendo as mesma contas para o país 2 obtenho: 


$$
\mathrm{PNB}_{2}=\mathrm{Y}_{2}+(\mathrm{P}-1)\left(\mathrm{N}_{2} \mathrm{X}_{2}+\mathrm{N}_{2} \mathrm{M}_{1}\right)
$$

pela óptica do dispêndio,

$$
\mathrm{PNB}_{2}=\mathrm{C}_{2}+\eta\left(\mathrm{dN} \mathrm{N}_{2} / \mathrm{dt}\right)-\mathrm{PN}_{1} \mathrm{M}_{2}-\mathrm{N}_{2} \mathrm{X}_{2}+(\mathrm{P}-1) \mathrm{N}_{2} \mathrm{M}_{1}
$$

e finalmente pela óptica da renda nacional,

$$
\mathrm{PNB}_{2}=\mathrm{w}_{2} \mathrm{~L}_{2}+\mathrm{mN}_{2}
$$

Alguns algebrismos são necessários para se encontrar as leis de movimento das variáveis de estado $\mathrm{N}_{1}$ e $\mathrm{N}_{2}$. Igualando-se as equações (1.15) e (1.16) temos que:

$$
\eta\left(d N_{1} / d t\right)=w_{1} L_{1}+m N_{1}-C_{1}-N_{2} X_{2}+(P-1) N_{2} M_{1}-P N_{1} M_{2}
$$

e igualando as equações (1.18) e (1.19) obtemos :

$$
-\mathrm{PN}_{1} \mathrm{M}_{2}=\mathrm{w}_{2} \mathrm{~L}_{2}+\mathrm{mN}_{2}-\mathrm{C}_{2}-\eta\left(d \mathrm{~N}_{2} / \mathrm{dt}\right)+\mathrm{N}_{2} \mathrm{X}_{2}-(\mathrm{P}-1) \mathrm{N}_{2} \mathrm{M}_{1}
$$

da substituição de (1.20) em (1.21) obtemos:

$$
\begin{aligned}
& \left(\mathrm{dN}_{1} / d t\right)=(1 / \eta)\left[w_{1} L_{1}+m N_{1}+w_{2} L_{2}+m N_{2}-C_{1}-C_{2}-\eta \gamma_{N 2} N_{2}\right] \\
& \left(d N_{2} / d t\right)=(1 / \eta)\left[w_{1} L_{1}+\eta N_{1}+w_{2} L_{2}+m N_{2}-C_{1}-C_{2}-\eta \gamma_{N 1} N_{1}\right]
\end{aligned}
$$

A equação (1.22) será a lei de movimento para a variável de estado $N_{1}$ e a equação (1.23) será a lei de movimento para a variável de estado $\mathrm{N}_{2}$. Note que a lei de movimento para o país 1 diz que o investimento em P\&D do pais 1 é igual a renda dos dois paises subtraída do consumo dos dois países menos o investimento no país 2 . $O$ fato é que a poupança conjunta dos dois paises é que irá financiar o investimento dos dois países sendo que o saldo da balança 
comercial é que irá transferir poupança de um país para outro de forma a cobrir a diferença entre poupança e investimento doméstico de cada país.

Para a resolução completa do modelo faz-se necessário a determinação da taxa de crescimento do consumo compatível com a maximização da utilidade dos agentes econômicos. O problema resultante das preferências dos agentes do país 1 é dado por:

$$
\operatorname{Max} \int_{0}^{+\infty} \log _{e}\left(C_{1}\right) e^{-p t} d t
$$

$$
\text { s. a: }\left(d N_{1} / d t\right)=(1 / \eta)\left[w_{1} L_{1}+m N_{1}+w_{2} L_{2}+m N_{2}-C_{1}-C_{2}-\eta \gamma_{N 2} N_{2}\right]
$$

Das condições de primeira ordem deste problema obtemos a taxa de crescimento do consumo:

$$
\gamma_{C 1}=r-\rho
$$

O problema do consumidor representativo no pais 2 leva a uma solução análoga.

Usando a taxa de retorno do país 1, que é a taxa internacional obtida na equação (1.13), a expressão de equilíbrio para a taxa de crescimento será dada por:

$$
\gamma_{C 1}=\left[\left(L_{1}+\phi^{1 /(1-\alpha)} L_{2}\right) / \eta\right]\left(A \alpha^{2}\right)^{1 /(1-\alpha)}\left(\alpha^{-1}-1\right)-\rho
$$

É imediato verificar que a taxa de crescimento do consumo será tanto maior quanto maior for o mercado do bloco, $\mathrm{L}_{1}+\mathrm{L}_{2}$, quanto menor for o custo de transporte ( maior $\phi$ ), e quanto menor for o custo de se produzir um novo insumo intermediário, $\eta$. Como a taxa de retorno é a mesma para os dois países temos $\gamma_{\mathrm{Cl}}=\gamma_{\mathrm{C} 2}$. Além disso, no estado estacionário consumo, produção e quantidade de insumos crescem a mesma taxa $\gamma$ dada pela equação (1.25). A demonstração deste fato é simples e pode ser encontrada em versão anterior deste artigo.

Comparando os resultados do modelo com integração aos resultados do modelo para o caso isolado é possível verificar que as taxas de crescimento são maiores após integração. No caso isolado a taxa de crescimento é dada por expressão análoga a (1.25) mas com $\mathrm{L}_{1}$ 
substituindo $L^{\text {" }}$, que é maior que $\mathrm{L}_{1}$. A intuição é que a integração promove um aumento do mercado de comercialização para os insumos intermediários e consequentemente um aumento da taxa de lucro. Chamando a taxa de crescimento de equilíbrio do caso isolado e de integração do pais 1 respectivamente de $\gamma_{1}^{\text {iwo }}$ e $\gamma_{1}{ }^{\text {int }}$ temos,

$$
\gamma_{1}^{i n t}=\left(1+\phi^{1 /(1-\alpha)} h^{-1}\right) \gamma_{1}^{i s 0}+\phi^{1 /(1-\alpha)} h^{-1} \rho
$$

onde $h=\left(L_{1} / L_{2}\right)$ denota um indice de vantagem comparativa em termos de extensão do mercado que é equivalente a um índice de vantagem comparativa em termos de dotação de mão-de-obra do país 1 relativamente ao país 2 . Obviamente haverá um ganho em termos de taxa de crescimento do $\operatorname{PNB}^{6}$ já que $\left(1+\phi^{1 /(1-a)} h^{-1}\right)>1$ e este ganho será tanto maior quanto menor for $h$. Além do mais, quanto menor for o custo de transporte (maior for $\phi$ ) maior será a taxa de crescimento, já que o efeito do custo de transporte é o de diminuir o mercado do outro país diminuindo as vendas de produtos produzidos no país 1 para o país 2 . Finalmente, o efeito da taxa de desconto intertemporal, $\rho$, é análogo ao exercido no caso da taxa de crescimento para o caso isolado, ou seja, uma maior taxa de desconto significa uma maior impaciência por poupar e consequentemente menor investimento em P\&D e menor taxa de crescimento.

Para o país 2 a taxa de retorno de integração mantém a seguinte relação com a taxa de crescimento para o caso isolado.

$$
\gamma_{2}^{\text {int }}=\left(h+\phi^{1 /(1-\alpha)}\right) \gamma_{1}^{i s o}+\left(h-1+\phi^{1 /(1-\alpha)}\right) \rho
$$

Por hipótese $h>1$ o que garante que $\gamma_{2}^{\text {int }}>\gamma_{1}{ }^{\text {iso }}$. Note que este aumento será tanto maior quanto maior for $\mathrm{h}$ (maior for o aumento do mercado com a integração) e quanto menor for o custo de transporte, já que um maior custo de transporte diminui as vendas das empresas produtoras de insumos intermediários que são de propriedade dos residentes do país 2 .

\footnotetext{
${ }^{6}$ A taxa de crescimento do PNB será igual a taxa de crescimento do nível de produção dado que o nível de diversidade de insumos, consumo e nível de produção crescem todos à mesma taxa dada pela equacăo (2.31).
} 
A integração também irá provocar uma variação instantânea no nivel de consumo. Primeiramente iremos analisar esta variação para o país 1. Pela identidade do produto e dada a igualdade entre (1.14) e (1.15), o consumo no país lserá dado por:

$$
C_{1}=Y_{1}-N_{1} X_{1}-(1+P) N_{1} M_{2}-(1+P) N_{2} X_{2}-N_{2} M_{1}-\eta \gamma_{N 1} N_{1}
$$

Utilizando as equações de demanda por insumos intermediários e levando em conta o fato de que os produtos importados pelo país 2 são os únicos que irão sofrer o custo de transporte, obtemos os valores de equilibrio para a quantidade de insumos utilizadas por cada país.

$$
\begin{array}{ll}
\mathrm{X}_{1}=\left(\mathrm{A} \alpha^{2}\right)^{1 /(1-\alpha)} \mathrm{L}_{1}, & \mathrm{M}_{1}=\left(\mathrm{A} \alpha^{2}\right)^{1 /(1-\alpha)} \mathrm{L}_{1}, \\
\mathrm{X}_{2}=\phi^{1 /(1-\alpha)}\left(\mathrm{A} \alpha^{2}\right)^{1 /(1-\alpha)} \mathrm{L}_{2}, & \mathrm{M}_{2}=\phi^{1 /(1-\alpha)}\left(\mathrm{A} \alpha^{2}\right)^{1 /(1-\alpha)} \mathrm{L}_{2}
\end{array}
$$

além do mais,

$$
\begin{aligned}
& Y_{1}=A L_{1}^{1-\alpha}\left(N_{1} X_{1}^{\alpha}+N_{2} M_{1}^{\alpha}\right)=\left(A \alpha^{2}\right)^{1 /(1-\alpha)} L_{1}\left(N_{1} \alpha^{-2}+N_{2} \alpha^{-2}\right) \\
& \eta \gamma N_{1}=\left(A \alpha^{2}\right)^{1 /(1-\alpha)}\left(\alpha^{-1}-1\right)\left(L_{1}+\phi^{1 /(1-\alpha)} L_{2}\right)-\rho \eta N_{1}
\end{aligned}
$$

Aplicando as equações acima na equação (1.28), podemos escrever o nivel de consumo inicial da integração como:

$$
\begin{aligned}
C_{1}{ }^{\text {int }}(0) & =\left(A \alpha^{2}\right)^{1 /(1-\alpha)}\left\{L_{1} N_{1}\left(\alpha^{-2}-\alpha^{-1}\right)+L_{1} N_{2}\left(\alpha^{-2}-1\right)-L_{2} N_{1}\left(2 \alpha^{-1} \phi^{1 /(1-\alpha)}\right)\right. \\
& \left.-L_{2} N_{2}\left(1+\alpha^{-1} \phi^{1 /(1-\alpha)}\right)\right\}+\rho \eta N_{1}
\end{aligned}
$$

Já o nível de consumo no caso isolado é dado por:

$$
C_{1}^{\text {iso }}(0)=\left(A \alpha^{2}\right)^{1 /(1-\alpha)} L_{1} N_{1}\left(\alpha^{-2}-\alpha^{-1}\right)+\rho \eta N_{1}
$$

Podemos observar que a integração terá um efeito ambíguo sobre o nível de consumo. Haverá um efeito positivo devido ao aumento do valor bruto da produção pela adição de 
insumos importados, representado pelo termo $\left(A \alpha^{2}\right)^{1 /(1-\alpha)} L_{1} N_{2} \alpha^{-2}$. No entanto, a integração diminuíra o consumo através de três canais. $O$ primeiro seria pelo incentivo a um maior investimento em P\&D ocasionado pelo aumento do mercado de comercialização dos insumos, representado por $-\left(A \alpha^{2}\right)^{1 /(1-\alpha)} L_{2} N_{1}\left(\alpha^{-1}-1\right) \phi^{1 /(1-\alpha)}$. O segundo canal de queda do consumo se daria pelo maior gasto em bens de consumo para a produção de bens intermediários devido a instalação das firmas produtoras de bens intermediários do país 2 no país 1 e pelo aumento dos gastos das firmas do próprio país 1 já que a demanda por insumos aumentou com a integração, efeito este dado por $-\left(A \alpha^{2}\right)^{1 /(1-\alpha)}\left[L_{1} N_{2}+L_{2} N_{1} \phi^{1 /(1-\alpha)}+L_{2} N_{2} \phi^{1 /(1-\alpha)}\right]$. Finalmente o terceiro e último canal se daria pelas exportações para o pais 2 surgidas com o fim do isolamento representado pelo termo $-\left(A \alpha^{2} \phi\right)^{1 /(1-\alpha)} \alpha^{-1}\left[L_{2} N_{1}+L_{2} N_{2}\right]$.

O efeito líquido da integração sobre o nível de consumo inicial irá depender do tamanho do mercado e da tecnologia do outro país, bem como do custo de transporte. No entanto, a análise que é mais relevante diz respeito a variação de utilidade do consumidor representativo levando-se em conta não só os efeitos sobre o consumo no momento da integração mas também a variação da taxa de crescimento do consumo devido a integração. A variação da utilidade intertemporal do consumidor representativo será considerada como variação do nível de bem-estar da economia. Este cálculo será feito na seção seguinte para os dois países. Antes disso, no entanto, iremos analisar a variação instantânea do nível de consumo para o país 2.

Fazendo-se contas análogas para o país 2 obtemos:

$$
\begin{aligned}
C_{2}{ }^{\text {int }}(0) & =\left(A \alpha^{2}\right)^{1 /(1-\alpha)}\left\{L_{2} N_{2}\left(\phi^{\alpha /(1-\alpha)} \alpha^{-2}-\phi^{1 /(1-\alpha)}\right)+L_{2} N_{1}\left(\phi^{\alpha /(1-\alpha)} \alpha^{-2}-\phi^{1 /(1-\alpha)} \alpha^{-1}\right)\right. \\
& \left.-L_{1} N_{2}\left(\alpha^{-1}-1\right)\right\}+\rho \eta N_{2}
\end{aligned}
$$

e na situação de isolamento,

$$
C_{2} \text { iso }(0)=\left(A \alpha^{2}\right)^{1 /(1-\alpha)} L_{2} N_{2}\left(\alpha^{-2}-\alpha^{-1}\right)+\rho \eta N_{2}
$$

Da mesma forma que para o pais com maior mercado, o efeito da integração sobre o nível de consumo será ambíguo. Por um lado o consumo tem uma tendência a aumentar já que o país 2 irá transferir suas empresas produtoras de bens intermediários para o pais 1 e 
consequentemente não irá gastar recursos para produzir insumos. Além disso, este fato faz com que o pais 2 importe do país 1 todos os seus insumos intermediários não exportando nada para o país 1, gerando assim uma poupança externa positiva que ajudará a financiar o investimento em P\&D e possibilitará o aumento do consumo doméstico. $O$ valor das importações é dado por $\left(A \alpha^{2}\right)^{1 /(1-\alpha)} \phi^{1 /(1-\alpha)} \alpha^{-1}\left(N_{2} L_{2}+N_{1} L_{1}\right)$. Outra forma na qual o consumo tende a aumentar é pelo aumento do valor bruto da produção devido a utilização de insumos importados. A tendência de queda do consumo surge do fato de que o aumento do mercado de comercialização dos insumos aumenta a taxa de retorno da descoberta de novos insumos, o que aumenta o investimento em P\&D e consequentemente diminui o nível de consumo.

O efeito do custo de transporte sobre o consumo inicial do país 2 também será ambíguo. Por um lado o consumo cairá por que o custo de transporte diminui a produtividade marginal dos insumos. Logo, como todos os insumos intermediários do país 2 serão importados, se empregará menor quantidade de insumos diminuindo assim o nível de produção e as importações, o que leva a uma queda do consumo inicial. Por outro lado, o consumo no país 2 crescerá com o aumento do custo de transporte pois este aumento provoca a queda da taxa de lucro da atividade de P\&D e portanto diminui o investimento, aumentando assim os recursos disponíveis para o consumo interno. $O$ resultado total de todos estes efeitos está representado na equação (1.30).

\section{III: Efeitos da integração sobre o bem-estar}

A variação do bem-estar com a integração irá depender da variação instantânea do nivel de consumo, da sua taxa de crescimento e da taxa de desconto intertemporal. Para analisarmos a variação da utilidade intertemporal é necessário comparar o nível de utilidade do agente representativo no caso isolado com o da integração. Iremos definir o ganho de bem-estar gerado pela integração como a diferença do nível de utilidade do indivíduo representativo do caso de integração para o caso isolado, ou seja:

$$
U_{1}^{\text {int }}-U_{1}^{\text {ino }}=\int_{0}^{\infty} \log _{e}\left[C_{1}{ }^{i n t}(t)\right] \exp (-\rho t) d t-\int_{0}^{\infty} \log _{e}\left[C_{1}^{i \infty 0}(t)\right] \exp (-\rho t) d t
$$




$$
\begin{aligned}
& =\int_{0}^{\infty} \log _{e}\left[\frac{C_{1}^{\text {int }}(0) \exp \left(\gamma_{1}^{\text {int } t}\right)}{C_{1}^{\text {iso }}(0) \exp \left(\gamma_{1}^{\text {ito } t} t\right)}\right] \exp (-\rho \mathrm{t}) \mathrm{dt} \\
& =\log _{e}\left[\frac{C_{1}^{\text {int }}(0)}{C_{1}^{\text {iso }}(0)}\right] \int_{0}^{\infty} \exp (-\rho \mathrm{t}) \mathrm{dt}+\left[\gamma_{1}^{\text {int }}-\gamma_{1}^{\text {iso }}\right] \int_{0}^{\infty} t \exp (-\rho \mathrm{t}) \mathrm{dt} \\
\mathrm{U}_{1}^{\text {int }}-\mathrm{U}_{1}^{\text {iso }} & =\frac{\log _{e}\left[C_{1}^{\text {int }}(0)\right]-\log _{e}\left[C_{1}^{\text {iso }}(0)\right]}{\rho}+\frac{\left[\gamma_{1}^{\text {int }}-\gamma_{1}^{\text {iso }}\right]}{\rho^{2}}
\end{aligned}
$$

Observe que a variação de bem-estar será composta de duas partes, uma correspondente a um efeito de curto prazo e a outra a um efeito de longo prazo. $O$ efeito de curto prazo é representado pela diferença das utilidades instantâneas do consumo logo no início da integração e pode ser positiva ou negativa. $O$ efeito de longo prazo, dado pela variação da taxa de crescimento do consumo, é sempre positivo já que a taxa de crescimento sempre aumenta com a integração.

$\mathrm{Na}$ comparação destes efeitos a taxa de desconto intertemporal tem um papel crucial pois quanto menor ela for maior será o peso dado ao efeito de longo prazo vis-a-vis o efeito de curto prazo. Por exemplo uma taxa de desconto intertemporal de 5\% daria um peso 20 vezes maior ao efeito de longo prazo relativamente ao efeito de curto prazo, enquanto que uma taxa de $3 \%$ daria um peso 33,33 vezes maior ao efeito de longo prazo. No entanto devemos notar que o efeito de curto prazo também dependerá desta taxa já que o consumo no caso isolado e em integração dependem da taxa de desconto intertemporal.

Abrem-se portanto duas possibilidades, dado que a taxa de crescimento do consumo sempre aumenta com a integração podemos ter um aumento ou uma queda do consumo inicial. No caso de ocorrer um aumento do consumo inicial estará garantido o ganho de bem-estar positivo já que o nível de consumo será maior em todo período de tempo após a integração. No caso do consumo inicial cair o ganho de bem estar poderá ser positivo ou negativo dependendo dos valores da queda do consumo e da taxa de desconto, pois como a taxa de crescimento em integração é sempre maior, a partir de algum ponto do tempo pós-integração o nível de consumo em integração passará a ser maior que o nível de consumo do caso isolado. As figuras 1 e 2 ilustram as duas situações. 


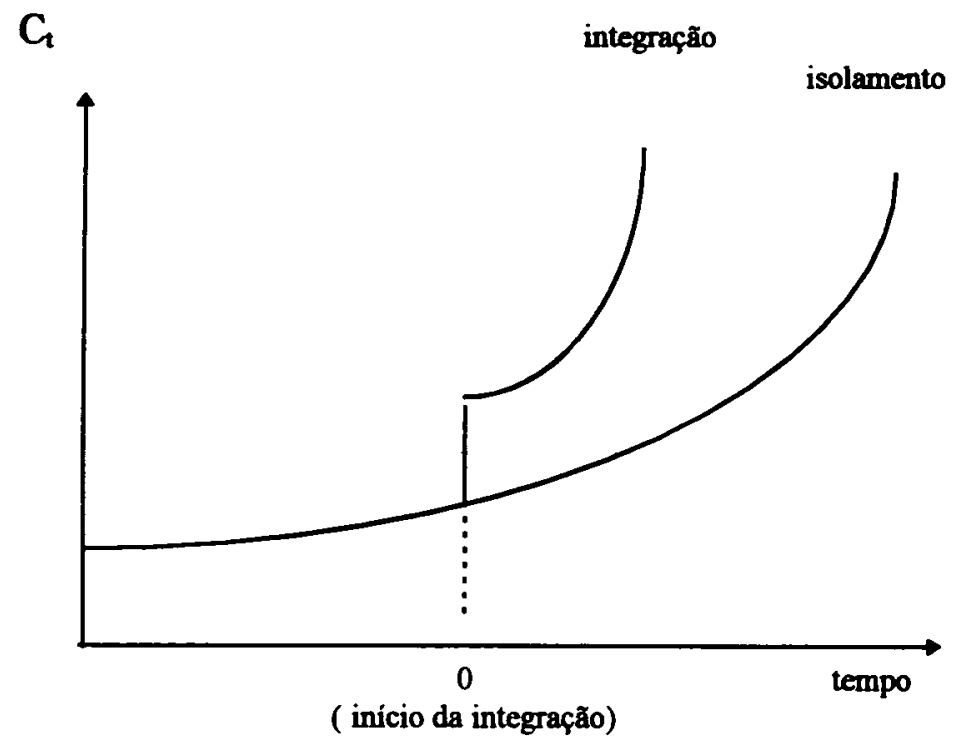

Figura 1 : Caso em que a integração promove um aumento instantâneo do nivel de consumo e onde fica garantido um ganho de bem-estar positivo

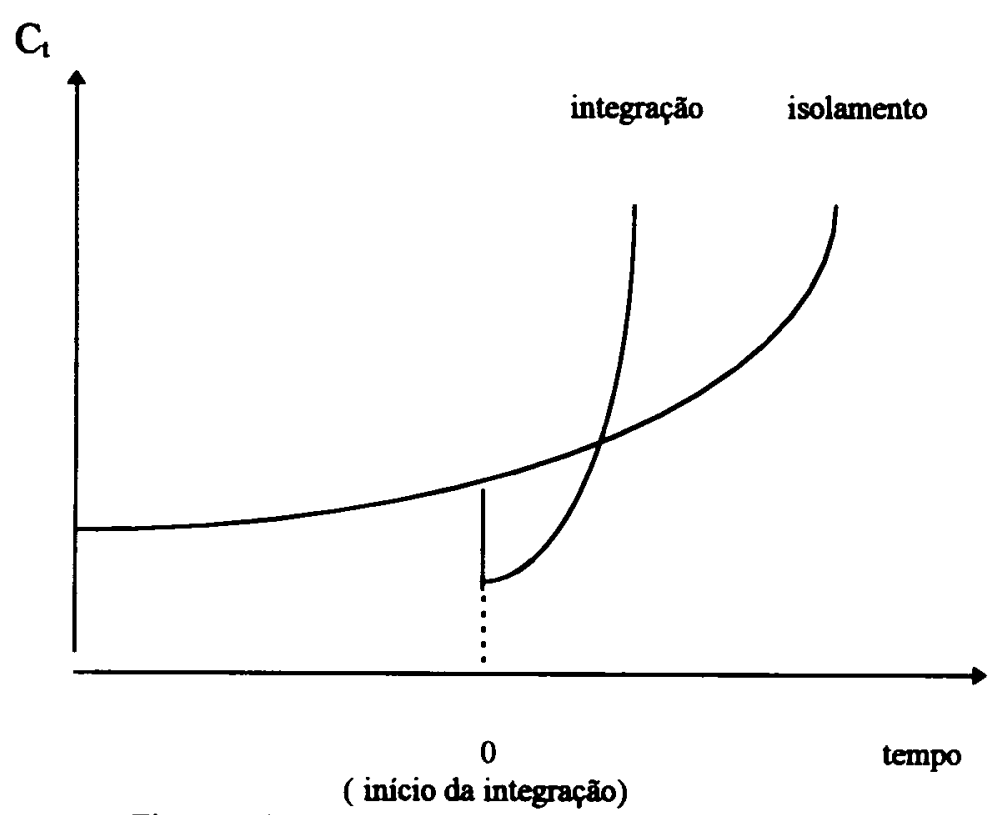

Figura 2: Caso em que a integração promove uma queda instantânea do nivel de consumo e onde o ganho de bem-estar irá depender da taxa de desconto intertemporal.

Como não é possível determinar se o nível de consumo inicial irá cair ou diminuir após a integração o ganho de bem estar não poderá ser determinado a priori. Na próxima seção apresentaremos uma simulação do modelo utilizando valores de parâmetros que correspondentes àqueles observados no Brasil e Argentina, de forma a tentar calcular ganhos 
de bem-estar. De qualquer forma é ainda possível analisar os efeitos de variações de parâmetros relevantes sobre o bem-estar, é isto que faremos a seguir.

Iremos considerar os efeitos sobre o bem-estar de um país integrado de variações dos parâmetros do outro país participante da integração, bem como do custo de transporte. Veremos primeiramente a estática comparativa para o país $1^{7}$. A partir das expressões encontradas na seção 2 e da equação (2.1) temos que:

$$
\begin{aligned}
& \mathrm{U}_{1}^{\text {int }}-\mathrm{U}_{1}^{\text {iso }}=\frac{1}{\rho} \log _{e}\left\{( A \alpha ^ { 2 } ) ^ { 1 / ( 1 - \alpha ) } \left[\mathrm{~L}_{1} \mathrm{~N}_{1}\left(\alpha^{-2}-\alpha^{-1}\right)+\mathrm{L}_{1} \mathrm{~N}_{2}\left(\alpha^{-2}-1\right)-\mathrm{L}_{2} \mathrm{~N}_{1}\left(2 \alpha^{-1} \phi^{1 /(1-\alpha)}\right)\right.\right. \\
& \left.\left.-\mathrm{L}_{2} \mathrm{~N}_{2}\left(1+\alpha^{-1} \phi^{1 /(1-\alpha)}\right)\right]+\rho \eta \mathrm{N}_{1}\right\}-\frac{1}{\rho} \log \left\{\left(A \alpha^{2}\right)^{1 /(1-\alpha)} \mathrm{L}_{1} \mathrm{~N}_{1}\left(\alpha^{-2}-\alpha^{-1}\right)+\rho \eta N_{1}\right\} \\
& +\frac{1}{\rho^{2}}\left[\left(\phi^{1 /(1-\alpha)} \mathrm{L}_{2}\right)\left(A \alpha^{2}\right)^{1 /(1-\alpha)}\left(\alpha^{-1}-1\right) / \eta\right]
\end{aligned}
$$

O efeito do nível de capital humano do país 2 sobre o ganho de bem estar do país 1 é dado pela equação abaixo,

$$
\frac{\partial\left(\mathrm{U}_{1}^{\mathrm{int}}-\mathrm{U}_{1}^{i s 0}\right)}{\partial \mathrm{L}_{2}}=\frac{\left(\mathrm{A} \alpha^{2} \phi\right)^{1 /(1-\alpha)}}{\rho}\left\{\frac{\left(\alpha^{-1}-1\right)}{\eta \rho}-\frac{\left[\mathrm{N}_{1}\left(2 \alpha^{-1)}\right)+\mathrm{N}_{2}\left(\alpha^{-1}+1\right)^{\prime}\right]}{\mathrm{C}_{1}{ }^{\mathrm{nt}}(0)}\right\},
$$

O efeito total sobre o bem estar será a soma de um efeito de curto prazo negativo e um efeito de longo prazo positivo. A parte com sinal negativo da equação (2.3) vem do fato de que um maior nível de capital humano no outro país integrado significa um maior mercado consumidor externo e consequentemente mais exportações, menor poupança externa, e também maiores lucros para o descobridor do insumo e portanto maior investimento em P\&D. 0 aumento de exportações e do investimento ocasiona, por sua vez, um menor consumo doméstico após a integração. A parte positiva do lado direito da equação (3.3) reflete o efeito de longo prazo da integração com um país com maior nível de capital humano, já que um maior mercado externo aumenta a taxa de crescimento do consumo.

\footnotetext{
'Observe que os resultados de estática comparativa desenvolvidos para a economia 1 só são válidos para valores de $L_{1}$ e $L_{2}$ tais que $L_{1}>L_{2}$.
} 
Podemos verificar entretanto que para valores suficientemente elevados do consumo inicial em integração e suficientemente pequenos do custo de pesquisa, o efeito final será positivo. Da mesma forma, se o nível tecnológico de ambos países $\left(\mathrm{N}_{1}\right.$ e $\left.\mathrm{N}_{2}\right)$ não for muito alto, haverá ganho de bem-estar com a integração.

O efeito de um aumento nível de tecnologia do país 2 (ou o efeito do país 1 se integrar com um país tecnologicamente mais avançado) é dado por:

$$
\frac{\partial\left(\mathrm{U}_{1}{ }^{\text {int }}-\mathrm{U}_{1}{ }^{\text {iso }}\right)}{\partial \mathrm{N}_{2}}=\frac{\left(\mathrm{A} \alpha^{2}\right)^{1 /(1-\alpha)}\left\{\mathrm{L}_{1}\left(\alpha^{-2}-1\right)-\mathrm{L}_{2}\left(\alpha^{-1}+1\right) \phi^{1 /(1-\alpha)}\right\}}{\rho \mathrm{C}_{1}{ }^{\text {int }}(0)}
$$

Se o nível de tecnologia do país 2 for maior, o efeito sobre a variação de utilidade do país 1 será todo de curto prazo, já que somente o consumo inicial é alterado. $\mathrm{O}$ sinal entretanto é ambíguo. Por um lado o consumo tende a aumentar devido a uma maior utilização de insumos intermediários, o que leva a um maior valor bruto da produção. Por outro lado, a tendência de queda se deve ao fato de que como as indústrias produtoras de bens intermediários de propriedade de residentes do pais 2 se localizam no país 1, um maior $\mathrm{N}_{2}$, implica em um maior número destas indústrias e consequentemente um maior nível de exportações para o país 2 ( já que estes insumos são produzidos em 1 e exportados para 2). 0 aumento das exportações, por sua vez, irá diminuir a poupança externa e para um dado nível de investimento doméstico retrair o consumo interno. Observe que se o nivel de capital humano do país 2 for relativamente pequeno teremos o efeito total positivo pois isto implicará em um pequeno aumento das exportações vis-a-vis um grande aumento do nível de produção.

Por fim o efeito do custo de transporte é dado pela equação abaixo.

$$
\frac{\partial\left(\mathrm{U}_{1}{ }^{\text {int }}-\mathrm{U}_{1}{ }^{i s o}\right)}{\partial \phi}=\frac{\left(\mathrm{A} \alpha^{2} \phi^{\alpha}\right)^{1 /(1-\alpha)} \mathrm{L}_{2}}{(1-\alpha) \rho}\left\{\frac{\left(\alpha^{-1}-1\right)}{\eta \rho}-\frac{\left[\mathrm{N}_{1}\left(2 \alpha^{-1}\right)+\mathrm{N}_{2}\left(\alpha^{-1}+1\right)\right]}{\mathrm{C}_{1}{ }^{\text {int }}(0)}\right\}
$$

Note que um maior $\phi$ implica em um menor custo de transporte dado que este custo é dado por $(1-\phi)$. $O$ efeito do custo de transporte também irá se dividir em um efeito de curto prazo negativo e de um efeito de longo prazo positivo. $O$ efeito negativo vem de que um 
menor custo de transporte aumenta as exportações e os investimentos em P\&D diminuindo, assim , o consumo. $\mathrm{O}$ efeito positivo decorre de que um menor custo de transporte implica em uma maior taxa de lucro da atividade de P\&D e portanto maior taxa de crescimento.

Passaremos agora a analisar os efeitos de variações dos parâmetros do país 1 sobre os ganhos de bem estar do país 2 decorrente da integração. A partir das equações da seção 2 e da equação (2.1) temos:

$$
\begin{aligned}
\mathrm{U}_{2}^{\text {int }}- & \mathrm{U}_{2}^{\mathrm{i}=0}=\frac{1}{\rho} \log _{e}\left\{( \mathrm { A } \alpha ^ { 2 } ) ^ { 1 / ( 1 - \alpha ) } \left[\mathrm{~L}_{2} \mathrm{~N}_{2}\left(\phi^{\alpha /(1-\alpha)} \alpha^{-2}+\phi^{1 /(1-\alpha)}\right)+\mathrm{L}_{2} \mathrm{~N}_{1}\left(\phi^{\alpha /(1-\alpha)} \alpha^{-2}+\right.\right.\right. \\
& \left.\left.\left.\phi^{1 /(1-\alpha)-\alpha)} \alpha^{-1}\right)-\mathrm{L}_{1} \mathrm{~N}_{2}\left(\alpha^{-1}-1\right)\right]+\rho \eta \mathrm{N}_{1}\right\}-\frac{1}{\rho} \log _{e}\left\{\left(A \alpha^{2}\right)^{1 /(1-\alpha)} \mathrm{L}_{2} \mathrm{~N}_{2}\left(\alpha^{-2}-\alpha^{-1}\right)+\rho \eta \mathrm{N}_{2}\right\} \\
& +\frac{1}{\rho^{2}}\left\{\left[\left(\phi^{1 /(1-\alpha)}-1\right) \mathrm{L}_{2}+\mathrm{L}_{1}\right]\left(\mathrm{A} \alpha^{2}\right)^{1 /(1-\alpha)}\left(\alpha^{-1}-1\right) \eta^{-1}\right\}
\end{aligned}
$$

A partir da equação (2.6) podemos analisar como mudanças nas dotações do país 1 e nos custos de transporte afetam o bem-estar da economia do país 2. Primeiramente com respeito ao nivel de capital humano da economia 1 temos:

$$
\frac{\partial\left(\mathrm{U}_{2}{ }^{\mathrm{int}}-\mathrm{U}_{2}^{i s o}\right)}{\partial \mathrm{L}_{1}}=\frac{\left(\mathrm{A} \alpha^{2}\right)^{1 /(1-\alpha)}\left(\alpha^{-1}-1\right)}{\rho}\left\{\frac{1}{\eta \rho}-\frac{\mathrm{N}_{2}}{\mathrm{C}_{2}{ }^{\text {int }}(0)}\right\}
$$

Novamente a situação envolve um trade-off entre perdas no curto prazo e ganhos no longo prazo, porque um maior mercado externo aumenta o investimento doméstico em P\&D, diminuindo o consumo inicial, mas aumenta a taxa de crescimento do consumo.

Com relação ao nível tecnológico do país 1 temos,

$$
\frac{\partial\left(\mathrm{U}_{2}^{\text {int }}-\mathrm{U}_{2}^{i s o}\right)}{\partial \mathrm{N}_{1}}=\frac{\left(\mathrm{A} \alpha^{2}\right)^{1 /(1-\alpha)} \mathrm{L}_{2}\left(\phi^{\alpha /(1-\alpha)} \alpha^{-2}+\phi^{1 /(1-\alpha)} \alpha^{-1}\right)}{\rho \mathrm{C}_{2}{ }^{\text {int }}(0)}>0
$$

Neste caso o efeito não é ambíguo. Um aumento do nível tecnológico do país 1 irá implicar em uma maior produção, pois se passará a utilizar mais insumos intermediários na 
função de produção e também aumentar-se-á o consumo pelo fato das importações aumentarem, o que por sua vez aumentará a poupança externa.

Por último tem-se o efeito do custo de transporte.

$$
\begin{aligned}
& \frac{\partial\left(\mathrm{U}_{2}{ }^{\text {int }}-\mathrm{U}_{2}{ }^{i s o}\right)}{\partial \phi}=\frac{\left(\mathrm{A} \alpha^{2} \phi^{\alpha}\right)^{1 /(1-\alpha)} \mathrm{L}_{2}}{(1-\alpha) \rho}+ \\
& \quad\left\{\frac{\left(\alpha^{-1}-1\right)}{\eta \rho}+\frac{\mathrm{N}_{2}\left(\phi^{2 \alpha-1} \alpha^{-1}+1\right)+\mathrm{N}_{1}\left(\phi^{2 \alpha-1} \alpha^{-1}+\alpha^{-1}\right)}{\mathrm{C}_{2}{ }^{\text {int }}(0)}\right\}>0
\end{aligned}
$$

O efeito portanto de uma queda do custo de transporte para o país 2 é de um aumento do bem-estar. Menor (1-ф) implica em maior importação, maior quantidade de insumos intermediários alocada na produção do bem de consumo final e também maior taxa de crescimento da economia devido ao aumento da taxa de retorno da atividade de P\&D.

Podemos, portanto, sumariar os resultados de estática comparativa da seguinte forma. No caso do país com maior mercado os efeitos serão sempre ambíguos. Um aumento do capital humano do outro pais ou uma queda do custo de transporte terá um o efeito de curto prazo negativo e um de longo prazo positivo. Portanto, para baixas taxas de desconto intertemporal o efeito positivo de longo prazo tenderá a prevalecer. $O$ aumento da tecnologia do pais com o menor estoque de capital humano também terá efeito indeterminado sobre o bem-estar do pais com maior estoque de capital humano. Contudo, se o mercado deste for suficientemente grande em relação ao pais de menor mercado o efeito positivo irá dominar.

Quanto ao país com menor mercado, o único efeito indeterminado é o de uma variação do capital humano do outro país, que se desdobrará em um efeito de curto prazo negativo e um de longo prazo positivo. Uma queda do custo de transporte ou um aumento da tecnologia do país com maior mercado sempre irá aumentar o nível de bem-estar do país com menor mercado. Desta forma, podemos concluir que um pais pequeno que desconte pouco o futuro terá um ganho de bem-estar positivo tanto maior quanto maior for o tamanho do outro país com o qual este está integrando. Note que esta última conclusão fortalece a idéia de que, se pensarmos a economia 1 como sendo o resto do mundo, é vantajoso para um país pequeno se abrir com relação ao resto do mundo desde que ele tenha como financiar seu déficit comercial. 
Dado que o nivel de consumo inicial e os efeitos de bem-estar irão depender de certa forma do nível tecnológico e da dotação de capital humano dos países envolvidos no processo de integração, realizaremos na próxima seção uma estimativa, através da simulação do modelo discutido, dos efeitos de uma integração entre Brasil e Argentina.

\section{IV: Integração, Brasil e Argentina.}

Nesta seção utilizamos os resultados da seção anterior para estimar de ganhos de bemestar bem como o efeito sobre crescimento de longo prazo, da integração entre Brasil e Argentina. $O$ interesse é mais ilustrativo do que propriamente preditivo, reconhecendo-se desde já que o modelo é apenas uma tentativa de aproximação da realidade.

O interesse pela integração Brasil-Argentina é motivada pelo fato destes países representarem conjuntamente $97,4 \%$ do PNB e $96,1 \%$ da população do Mercosul segundo dados do Banco Mundial para o ano de 1992. Desta forma o efeito do Mercosul sobre ambos os países é praticamente o mesmo de uma integração isolada entre Brasil e Argentina

A estimativa irá depender de alguns parâmetros que necessitaremos calcular, a saber, a participação do capital na renda, $\alpha$, o custo de transporte, (1- $\phi)$, o nível de capital humano, $L$, a diversidade de insumos ou complexidade tecnológica, $\mathrm{N}$, e o custo de aumento da tecnologia, $\eta$.

O custo de transporte entre Brasil e Argentina foi obtido através de planilhas de custo fornecidas por uma empresa de comércio exterior ${ }^{8}$. Estes custos compreendem todos os custos de se importar ou exportar uma mercadoria excluindo-se os impostos: frete, seguro, despesas portuárias, etc. $\mathrm{O}$ resultado a que se chegou foi de um custo de transporte aproximado de $21 \%$ para o transporte rodoviário e de $17 \%$ para o transporte marítimo. Optamos, portanto, trabalhar com $\phi=0,8$, que reflete um custo intermediário de $20 \%$ entre o transporte marítimo e o rodoviário.

A participação do capital na renda para Brasil e Argentina foi extraída de um estudo feito por Elias (1990) sobre fontes de crescimento para sete países latino americanos, entre eles Brasil e Argentina. Os resultados obtidos indicam participações do capital na renda de $62,9 \%$

\footnotetext{
${ }^{8}$ As planilhas de custo estão discriminadas no apêndice de uma versão anterior deste artigo.
} 
para a Argentina e de 62,1\% para o Brasil. Para efeito de cálculo iremos trabalhar com $\alpha=0,62$. No entanto, este valor parece bastante alto em relação aos países desenvolvidos que apresentam uma participação do capital na renda em torno de $35 \%$. Desta forma , supondo ainda que Brasil e Argentina possuem valores mais altos que os paises desenvolvidos, iremos trabalhar também $\operatorname{com} \alpha=0,5$.

Os outro parâmetros $\mathrm{L}, \mathrm{N}$ e $\eta$ serão estimados a partir dos seguintes dados disponíveis para o ano de 1992. 
TABELA 1

\begin{tabular}{c|c|c|c|c|c|c}
\hline País & $\begin{array}{c}\text { Popul. } \\
\text { (milhóes) } \\
(1992)\end{array}$ & $\begin{array}{c}\text { PNB per } \\
\text { capita } \\
\text { (USS/92) }\end{array}$ & $\begin{array}{c}\text { PPPY per } \\
\text { capita (USA } \\
=100)\end{array}$ & $\begin{array}{c}\text { Anos de } \\
\text { estudo per } \\
\text { capita }^{b}\end{array}$ & $\begin{array}{c}\text { Popul. } \\
\text { Economic } \\
\text { Ativa } \\
\text { (milhres) }\end{array}$ & $\begin{array}{c}\text { Taxa de } \\
\text { cresc. PNB } \\
\text { per capita } \\
\text { (65/89) }\end{array}$ \\
\hline Brasil & 153,9 & 2.770 & 22,7 & 3,5 & 95 & $3,5 \%$ a.a. \\
\hline Argentina & 33,1 & 6.050 & 26,3 & 6,7 & 20 & $-0,1 \%$ a.a. \\
\hline
\end{tabular}

Fonte: Relatório do Banco Mundial (1992), (1990).

Notas: a: Power Purchase Parity Income, é uma medida de renda per capita que leva em conta a diferença de preços relativos entre os paises.

b: Dados paro o ano de 1985 segundo a base de dados Barro \& Lee(1993)

Para evitar diferenças de preços relativos iremos considerar a renda em termos de paridade de poder de compra (PPP). Sendo $\mathrm{Y}_{\mathrm{A}}$ e $\mathrm{Y}_{\mathrm{B}}$ as rendas totais de Argentina e Brasil em PPP tenho:

$$
\begin{aligned}
& Y=P P P Y \times \text { População } \\
& Y_{A}=26,3 \times 33,1=870,53 \\
& Y_{B}=22,7 \times 153,9=3493,53
\end{aligned}
$$

O nível de capital humano para cada país foi obtido através da multiplicação da escolaridade média ( anos completos de estudo per capita ), pelo número total de habitantes que compõem a população economicamente ativa (PEA). $\mathbf{O}$ nível de capital humano será representado portanto pelo número total de anos de estudo da PEA. Chamando de $L_{A}$ e $L_{B} 0$ nivel de capital humano de Argentina e Brasil respectivamente tenho?:

$$
\begin{aligned}
& \mathrm{L}_{A}=20 \times 6,7=134 \\
& \mathrm{~L}_{B}=95 \times 3,5=332,5
\end{aligned}
$$

${ }^{9}$ Observe que os valores absolutos de L, $\mathrm{N}$ e $\eta$ não são relevantes, $\mathrm{O}$ interesse aqui está nos valores relativos. 
O nível de diversidade de insumos ou complexidade tecnológica será obtido de forma residual. Dado que a renda de equilíbrio no caso isolado ${ }^{10}$ é dada por $Y=\left(A \alpha^{2}\right)^{1 /(1-\alpha)} \alpha^{-2} L N$, e normalizando o valor de $A$ de forma que $A=1$, temos:

$$
N=Y /\left(\alpha^{2 \alpha /(1-\alpha)} L\right)
$$

O valor para o custo de pesquisa será obtido através da equação da taxa de crescimento para o caso isolado, e que é dado por:

$$
\gamma=\left[\left(A \alpha^{2}\right)^{1 /(1-\alpha)}\left(\alpha^{-1}-1\right) L / \eta\right]-\rho
$$

É imediato verificar que:

$$
\eta=\left[\alpha^{2 /(1-\alpha)}\left(\alpha^{-1}-1\right) L\right] /(\gamma+\rho)
$$

Note que a taxa de crescimento per capita ao ano da Argentina foi de aproximadamente zero $(-0,1 \%)$ enquanto que para o Brasil tivemos uma taxa de 3,5\%. Desta forma, o valor de $\eta$ deverá satisfazer as duas condições que se seguem:

$$
\begin{aligned}
& \eta>\left[\alpha^{2 /(1-\alpha)}\left(\alpha^{-1}-1\right) L_{A}\right] / \rho \\
& \eta=\left[\alpha^{2 /(1-\alpha)}\left(\alpha^{-1}-1\right) L_{B}\right] /(0,035+\rho)
\end{aligned}
$$

Através dos valores da tabela acima e das equações desta seção e das seções 2 e 3 posso estimar os ganhos de bem-estar decorrentes da integração entre Brasil e Argentina de acordo com as equações desenvolvidas na seção 2 . Note que o modelo prevê que as indústrias produtoras de bens intermediários que possuem ganhos de escala irão concentrar sua localização no Brasil dados os custos de transporte e o fato do Brasil concentrar um maior

\footnotetext{
${ }^{10} \mathrm{~A}$ suposição de que Brasil e Argentina são economias isoladas não é muito irrealista na medida em que estas cconomias possuem um grau de abertura relativamente pequeno em relaça a outros países. Segundo dados do Banco Mundial as exportaçōes do Brasil representaram 10\% do PNB brasileiro e as da Argentina 7\% do PNB argentino para o ano de 1992.
} 
mercado consumidor destes insumos. Portanto, a Argentina será a economia 2 e o Brasil a economia 1. Serão feitas quatro simulações usando-se taxas de desconto intertemporal de $3 \%$ e $5 \%$ e participações do capital na renda de $50 \%$ e $62 \%$. Procedendo os cálculos ${ }^{11}$ para consumo taxa de crescimento e variação do bem-estar temos:

TABELA 2

Estimativas para a Integraçio Brasil-Argentina

\begin{tabular}{|c|c|c|c|c|}
\hline & $\alpha=0,5 \quad \rho=0,03$ & $\alpha=0,5 \rho=0,05$ & $\alpha=0,62 \rho=0,03$ & $\alpha=0,62 \rho=0,05$ \\
\hline$\gamma^{\text {int }}$ (ao ano) & $\overline{5,2 \%}$ & $5,7 \%$ & $5,0 \%$ & $5,4 \%$ \\
\hline$C_{\operatorname{Bn}}=0$ & 2150,3 & 2261,0 & $\overline{1708,6}$ & $\overline{1812,9}$ \\
\hline $\mathrm{C}_{\mathrm{Br}} \mathrm{s}^{\mathrm{nt}}$ & 2448,1 & 2558,8 & 1586,6 & $\overline{1691,0}$ \\
\hline$\left(U_{B r e}{ }^{i n t}-U_{B r e}{ }^{309}\right)$ & $23,21>0$ & $11,27>0$ & $14,19>0$ & $6,21>0$ \\
\hline $\mathrm{C}_{\mathrm{AB}_{\mathrm{B}}}$ & 653,2 & 653,2 & 535,8 & 535,8 \\
\hline $\mathrm{C}_{\mathrm{Arg}}^{\text {int }}$ & 943,9 & 1012,2 & 639,15 & 703,5 \\
\hline$\left(U_{A v g}^{i n t}-U_{A r g}^{i s o}\right)$ & $70,04>0$ & $31,55>0$ & $61,43>0$ & $27,05>0$ \\
\hline
\end{tabular}

Quando a participação do capital na renda é de 50\% há um ganho de bem-estar positivo tanto no curto prazo quanto no longo prazo para Brasil e Argentina, com taxas de crescimento de $5,2 \%$ (quando $\rho=0,03$ ) e $5,7 \%$ (quando $\rho=0,05$ ). $O$ fato do ganho Argentino ser mais elevado é justificado pelo fato de que com a integração ambos os paises crescem a mesma taxa, mas no entanto a Argentina parte de uma taxa de crescimento zero enquanto que o Brasil parte de $3,5 \%$.

Quando a participação do capital na renda é de $62 \%$ a taxa de crescimento estimada para ambos os países e de $5,0 \%$ (quando $\rho=0,03$ ) e $5,4 \%$ (quando $\rho=0,05$ ). A única mudança qualitativa observada é que o Brasil passaria a sofrer uma queda no nivel de consumo logo após a integração, ocasionando desta forma uma perda de bem-estar no curto prazo para o Brasil. Isto por que, como o Brasil é o país com maior mercado, ele investirá mais na produção de insumos e também terá um superávit na balança comercial, tendo, portanto, que realizar um

\footnotetext{
${ }^{11}$ No caso da Argentina seu consumo em isolamento não coresponderá a fórmula obtida para $C_{2}{ }^{\text {io }}(0)$, equação (2.44) devido ao fato que em isolamento a Argentina não investia em P\&D. A expressão para o consumo fica sendo $C_{2}{ }^{m 0}(0)=\left(A \alpha^{2}\right)^{1 /(1-\alpha)} L_{2} N_{2}\left(\alpha^{-2}-1\right)$
} 
maior sacrificio de consumo presente relativamente à Argentina. Sacrificio este que será tanto maior quanto maior for a participação do capital na renda. Portanto, para uma participação do capital na renda mais alta, no caso $62 \%$, o consumo inicial irá cair.

Com relação ao ganho de bem-estar com a integração a estimativa nos diz que estes serão sempre positivos para ambos países, sendo que a perda de bem estar que o Brasil sofre no curto prazo é compensada pelo ganho de longo prazo. Isto é, apesar do nivel de consumo no Brasil ter caído com a integração, ele passa a crescer mais rápido e em algum tempo irá superar o consumo de isolamento: para $\alpha=0,62$ e $\rho=0,03$ isto levaria 4,94 anos e para $\alpha=0,62$ e $\rho=0,05$ o tempo seria de 3,66 anos.

\section{Seção 5: Comentários Finais}

A idéia de que o comércio internacional sem restrições tende a promover o crescimento do produto e do consumo é confirmada pelos resultados do modelo. No entanto, como procuramos enfatizar através dos cálculos de variação do bem-estar, maior crescimento não significa necessariamente maior satisfação por parte dos agentes desta economia, na medida em que crescimento maior implica em um maior nível de investimento e em um maior sacrificio de consumo inicial por parte dos agentes econômicos.

De acordo com o modelo, no entanto, mesmo com um maior nível de investimento ainda é possível aumentar o nível de consumo no curto prazo. A integração sempre promove um aumento do valor da produção devido a utilização de novos insumos que a economia isolada não tinha acesso até então. Estes novos insumos importados provocam um aumento da produtividade da mão-de-obra gerando, assim, um maior nível de produção. Desta forma é possível conciliar um aumento simultâneo do investimento e do consumo. Neste caso, estaria garantido um ganho de bem-estar positivo para qualquer taxa de desconto intertemporal dos agentes desta economia já que o nível e a taxa de crescimento do consumo aumentariam.

Outro aspecto explorado pelo modelo diz respeito ao custo de transporte. Defendeu-se não apenas a idéia de que o custo de transporte tem efeitos de desincentivo ao comércio internacional, diminuindo o volume de bens comercializados, mas também que tal custo tem um efeito na localização da produção, concentrando-a próxima ao maior mercado. Apesar das 
hipóteses serem bem restritivas, em termos de opções de escolha de localização e de que ganhos de escala implicam na utilização de apenas uma firma para produzir um determinado insumo, este resultado não parece incoerente com o fato de se observar uma grande concentração industrial junto aos grandes centros populacionais.

Em relação a estimativa de ganhos de bem-estar para o Brasil e Argentina podemos fazer algumas ressalvas. Primeiramente devido ao fato do modelo não supor nenhuma restrição ao financiamento de déficits comerciais, não parece viável que a Argentina mantenha um saldo comercial negativo com o Brasil durante muito tempo pelos simples fato de se ver impossibilitada de financia-lo. Outra ressalva diz respeito a diferente estrutura tributária e de custos de cada país que poderia alterar a localização das indústrias de bens intermediários, i. e., diferenças de tributação e de custos também iriam afetar a localização das indústrias. No entanto, procurou-se ressaltar com a estimativa que o efeito isolado do custo de transporte e do tamanho do mercado tende a concentrar o investimento em novas fäbricas no Brasil vis-a-vis a Argentina.

A estimativa realizada forneceu um aumento da taxa de crescimento do produto per capita de ambos os países para algo em torno de 5\% ao ano. Além do mais, estima-se um ganho maior de bem-estar para o lado argentino uma vez que a variação da taxa de crescimento foi maior para a este país e que o seu nível de consumo teve um ganho instantâneo devido ao aumento do nível da produção e do déficit comercial com o Brasil. Note também, que se pensarmos a economia 1 (a major economia) como sendo o resto do mundo, a conclusão do modelo é que é vantajoso para um pais pequeno se abrir com relação ao resto do mundo desde que ele tenha como financiar seu déficit comercial. Ou que, quanto maior o bloco em que ela se integra, maior os ganhos de bem-estar e crescimento e que, como mostram as simulações do modelo, os ganhos do livre comércio superam em muito as vantagens de autarquia.

\section{Apêndice : Custos de Transporte Brasil Argentina}

A estimativa de custo de transporte para o comércio internacional entre Brasil e Argentina foi baseada nas planilhas de custo da empresa de comércio exterior H.H. Picchioni. 
Seguem abaixo tais planilhas para o mês de julho de 1996. 
Custo de Transporte Marítimo - Argentina/Brasil (RS) (Rio de Janeiro/Buenos Aires)

\begin{tabular}{c|c|c|c}
\hline Bem & Automóvel & $\begin{array}{r}\text { Tecido algodão } \\
(25.000 \mathrm{Kg})\end{array}$ & Feijão (24.00Kg) \\
\hline Custos & & & \\
\hline Valor FOB & 16.000 & 16.000 & 16.000 \\
\hline Frete & 1.200 & 1.200 & 1.200 \\
\hline Seguro & 270 & 270 & 270 \\
\hline Emolumento & 70 & 70 & 70 \\
\hline Desp. Banco & 500 & 500 & 500 \\
\hline Desp. Portos & 1.160 & 1.160 & 1.160 \\
\hline Total & 19.200 & 19.200 & 19.200 \\
\hline $\begin{array}{c}\text { Custo de } \\
\text { transporte }\end{array}$ & 3.200 & 3.200 & 3.200 \\
\hline (1- $\phi)$ & & & 0,17 \\
\hline
\end{tabular}

Custo de Transporte Rodoviário-Argentina/Brasil (RS) (Rio de Janeiro/Buenos Aires)

\begin{tabular}{l|c|c|c}
\hline \multicolumn{1}{|c|}{ Bem } & Automóvel & $\begin{array}{c}\text { Tecido algodăo } \\
(25.000 \mathrm{Kg})\end{array}$ & Feijão (24.00Kg) \\
\hline Valor FOB & 16.000 & 16.000 & 16.000 \\
\hline Frete & 3.000 & 3.000 & 3.000 \\
\hline Seguro & 270 & 200 & 200 \\
\hline $\begin{array}{l}\text { Despesas } \\
\text { emolumento }\end{array}$ & 70 & 70 & 70 \\
\hline $\begin{array}{l}\text { Despesas } \\
\text { bancárias }\end{array}$ & 500 & 500 & 500 \\
\hline $\begin{array}{l}\text { Alfândega } \\
\text { Terrestre }\end{array}$ & 530 & 530 & 530 \\
\hline Total & & & \\
\hline $\begin{array}{l}\text { Custo de } \\
\text { transporte }\end{array}$ & 20.370 & 20.300 & 20.300 \\
\hline (1-ф) & 4.370 & 4.300 & 4.300 \\
\hline
\end{tabular}

- Para o porto de Nova York este custo seria de R\$675,00, ou seja , $42 \%$ menor, levando a um custo de transporte de $14,5 \%$, i.e., $(1-\phi)=14,5$. 


\section{Referências:}

Aghion, Philippe \& Howitt, Peter - " A Model of Growth Through Creative Destruction", Econometrica, Vol. 60, n² March, 1992, 323-351.

Barro, Robert J. \& Lee, Jong Wha - "International Comparisons of Educational Attainment" , NBER Working Paper N 4394, 1993.

Barro, Robert J. \& Sala-i-Martin, Xavier - “ Economic Growth” , Mc Graw Hill Inc., San Francisco, 1995.

Cass, David - " Optimum Growth in an Aggregative Model of Capital Accumulation” Review of Economics Studies, 32 July, 1965, 233-240

Elias, Jose V. - "Sources of Growth - a study of seven latin american economies" - Executive Sumary, San Francisco: ICS Press/ ICEG, 1992.

Greenaway, David - "Policy Forum Regionalism in the World Economy: Editorial Note” The Economic Journal, 102 Nov. 1992, 1488-1499.

Grossman, Gene M. \& Helpman, Elhanan - "Quality Ladders in the Theory of Growth" The Review of Economic Studies, 1991.

Koopmans, Tjalling C. - " On the Concept of of Optimal Economic Growth" - in the Econometric Approach to Development Planning, Amsterdam, North Holland, 1965.

Riviera-Batiz, Luis A. \& Xie, Danyang - “ Integration Among Unequals” - Regional Science and Urban Economics, 23, 1993, 337-354.

Romer, Paul M. - "Endogenous Technological Change" - Journal of Political Economy, Vol. 98 n $^{\circ} 5$ pt. 2, 1990, s71-s101.

Romer, Paul M. - "The Origins of Economic Growth" - Journal of Economic Perspectives Vol. $8 n^{\circ} 1$ Winter, 1994, 3-22.

Ruffin, Roy J. - "Endogenous Growth and International Trade" - Federal Reserve Bank of Dallas, Research Paper $n^{\circ}$ 9332, Aug. 1993.

Sapir, André - "Regional Integration in Europe" - The Economic Journal, 102 Nov, 1992, 1491-1506.

Solow Robert M. - " Technical Change and the Aggregate Production Function" - Review of Economics and Statistics, 39 (August), 1957, 312-320 


\section{ENSAIOS ECONÔMICOS DA EPGE}

200. A VISÃO TEÓRICA SOBRE MODELOS PREVIDENCIÁRIOS: O CASO BRASILEIRO Luiz Guilherme Schymura de Oliveira - Outubro de 1992 - 23 pág. (esgotado)

201. HIPERINFLAÇÃO: CÂMBIO, MOEDA E ÂNCORAS NOMINAIS - Fernando de Holanda Barbosa - Novembro de 1992 - 10 pág. (esgotado)

202. PREVIDÊNCIA SOCIAL: CIDADANIA E PROVISÃO - Clovis de Faro - Novembro de 1992 - 31 pág. (esgotado)

203. OS BANCOS ESTADUAIS E O DESCONTROLE FISCAL: ALGUNS ASPECTOS Sérgio Ribeiro da Costa Werlang e Armínio Fraga Neto - Novembro de 1992 - 24 pág. (esgotado)

204. TEORIAS ECONÔMICAS: A MEIA-VERDADE TEMPORÁRIA - Antonio Maria da Silveira - Dezembro de 1992 - 36 pág. (esgotado)

205. THE RICARDIAN VICE AND THE INDETERMINATION OF SENIOR - Antonio Maria da Silveira - Dezembro de 1992 - 35 pág. (esgotado)

206. HIPERINFLAÇÃO E A FORMA FUNCIONAL DA EQUAÇÃO DE DEMANDA DE MOEDA - Fernando de Holanda Barbosa - Janeiro de 1993 - 27 pág. (esgotado)

207. REFORMA FINANCEIRA - ASPECTOS GERAIS E ANÁLISE DO PROJETO DA LEI COMPLEMENTAR - Rubens Penha Cysne - fevereiro de 1993 - 37 pág. (esgotado)

208. ABUSO ECONÔMICO E O CASO DA LEI 8.002 - Luiz Guilherme Schymura de Oliveira e Sérgio Ribeiro da Costa Werlang - fevereiro de 1993 - 18 pág. (esgotado)

209. ELEMENTOS DE UMA ESTRATÉGIA PARA O DESENVOLVIMENTO DA AGRICULTURA BRASILEIRA - Antonio Salazar Pessoa Brandão e Eliseu Alves Fevereiro de 1993 - 370pág. (esgotado)

210. PREVIDÊNCIA SOCIAL PÚBLICA: A EXPERIÊNCIA BRASILEIRA - Hélio Portocarrero de Castro, Luiz Guilherme Schymura de Oliveira, Renato Fragelli Cardoso e Uriel de Magalhães - Março de 1993 - 35 pág - (esgotado) .

211. OS SISTEMAS PREVIDENCIÁRIOS E UMA PROPOSTA PARA A REFORMULACAO DO MODELO BRASILEIRO - Helio Portocarrero de Castro, Luiz Guilherme Schymura de Oliveira, Renato Fragelli Cardoso e Uriel de Magalhães - Março de 1993 - 43 pág. (esgotado)

212. THE INDETERMINATION OF SENIOR (OR THE INDETERMINATION OF WAGNER) AND SCHMOLLER AS A SOCIAL ECONOMIST - Antonio Maria da Silveira - Março de 1993 - 29 pág. (esgotado)

213. NASH EQUILIBRIUM UNDER KNIGHTIAN UNCERTAINTY: BREAKING DOWN BACKWARD INDUCTION (Extensively Revised Version) - James Dow e Sérgio Ribeiro da Costa Werlang - Abril de 199336 pág. (esgotado)

214. ON THE DIFFERENTIABILITY OF THE CONSUMER DEMAND FUNCTION - Paulo Klinger Monteiro, Mário Rui Páscoa e Sérgio Ribeiro da Costa Werlang - Maio de 1993 19 pág. (esgotado) 
215. DETERMINAÇÃO DE PREÇOS DE ATIVOS, ARBITRAGEM, MERCADO A TERMO E MERCADO FUTURO - Sérgio Ribeiro da Costa Werlang e Flávio Auler - Agosto de 1993 69 pág. (esgotado).

216. SISTEMA MONETÁRIO VERSÃO REVISADA - Mario Henrique Simonsen e Rubens Penha Cysne - Agosto de 1993 - 69 pág. (esgotado).

217. CAIXAS DE CONVERSÃO - Fernando Antônio Hadba - Agosto de 1993 - 28 pág.

218. A ECONOMIA BRASILEIRA NO PERÍODO MILITAR - Rubens Penha Cysne - Agosto de 1993 - 50 pág. (esgotado).

219. IMPÔSTO INFLACIONÁRIO E TRANSFERÊNCIAS INFLACIONÁRIAS - Rubens Penha Cysne - Agosto de 1993 - 14 pág. (esgotado).

220. PREVISÕES DE M1 COM DADOS MENSAIS - Rubens Penha Cysne e João Victor Issler Setembro de 1993 - 20 pág. (esgotado)

221. TOPOLOGIA E CÁlCULO NO Rn - Rubens Penha Cysne e Humberto Moreira Setembro de 1993 - 106 pág. (esgotado)

222. EMPRÉSTIMOS DE MÉDIO E LONGO PRAZOS E INFLAÇÃO: A QUESTÃO DA INDEXAÇÃO - Clovis de Faro - Outubro de 1993 - 23 pág.

223. ESTUDOS SOBRE A INDETERMINAÇÃO DE SENIOR, vol. 1 - Nelson H. Barbosa, Fábio N.P. Freitas, Carlos F.L.R. Lopes, Marcos B. Monteiro, Antonio Maria da Silveira (Coordenador) e Matias Vernengo - Outubro de 1993 - 249 pág (esgotado)

224. A SUBSTITUIÇÃO DE MOEDA NO BRASIL: A MOEDA INDEXADA - Fernando de Holanda Barbosa e Pedro Luiz Valls Pereira - Novembro de 1993 - 23 pág.

225. FINANCIAL INTEGRATION AND PUBLIC FINANCIAL INSTITUTIONS - Walter Novaes e Sérgio Ribeiro da Costa Werlang - Novembro de 1993 - 29 pág

226. LAWS OF LARGE NUMBERS FOR NON-ADDITIVE PROBABILITIES - James Dow e Sérgio Ribeiro da Costa Werlang - Dezembro de 1993 - 26 pág.

227. A ECONOMIA BRASILEIRA NO PERÍODO MILITAR - VERSÃO REVISADA - Rubens Penha Cysne - Janeiro de 1994 - 45 pág. (esgotado)

228. THE IMPACT OF PUBLIC CAPITAL AND PUBLIC INVESTMENT ON ECONOMIC GROWTH: AN EMPIRICAL INVESTIGATION - Pedro Cavalcanti Ferreira - Fevereiro de 1994 - 37 pág. (esgotado)

229. FROM THE BRAZILIAN PAY AS YOU GO PENSION SYSTEM TO CAPITALIZATION: BAILING OUT THE GOVERNMENT - José Luiz de Carvalho e Clóvis de Faro - Fevereiro de 1994 - 24 pág.

230. ESTUDOS SOBRE A INDETERMINAÇÃO DE SENIOR - vol. II - Brena Paula Magno Fernandez, Maria Tereza Garcia Duarte, Sergio Grumbach, Antonio Maria da Silveira (Coordenador) - Fevereiro de 1994 - 51 pág.(esgotado)

231. ESTABILIZAÇÃO DE PREÇOS AGRÍCOLAS NO BRASIL: AVALIAÇÃO E PERSPECTIVAS - Clovis de Faro e José Luiz Carvalho - Março de 1994 - 33 pág. (esgotado)

232. ESTIMATING SECTORAL CYCLES USING COINTEGRATION AND COMMON FEATURES - Robert F. Engle e João Victor Issler - Março de 1994 - 55 pág. (esgotado) 
233. COMMON CYCLES IN MACROECONOMIC AGGREGATES - João Victor Issler e Farshid Vahid - Abril de 1994 - 60 pág.

234. BANDAS DE CÂMBIO: TEORIA, EVIDÊNCIA EMPÍRICA E SUA POSSÍVEL APLICAÇÃO NO BRASIL - Aloisio Pessoa de Araújo e Cypriano Lopes Feijó Filho - Abril de 1994 - 98 pág. (esgotado)

235. O HEDGE DA DÍvidA EXTERNA BRASILEIRA - Aloisio Pessoa de Araújo, Túlio Luz Barbosa, Amélia de Fátima F. Semblano e Maria Haydée Morales - Abril de 1994 - 109 pág. (esgotado)

236. TESTING THE EXTERNALITIES HYPOTHESIS OF ENDOGENOUS GROWTH USING COINTEGRATION - Pedro Cavalcanti Ferreira e João Victor Issler - Abril de 1994 - 37 pág. (esgotado)

237. THE BRAZILIAN SOCIAL SECURITY PROGRAM: DIAGNOSIS AND PROPOSAL FOR REFORM - Renato Fragelli; Uriel de Magalhães; Helio Portocarrero e Luiz Guilherme Schymura - Maio de 1994 - 32 pág.

238. REGIMES COMPLEMENTARES DE PREVIDÊNCIA - Hélio de Oliveira Portocarrero de Castro, Luiz Guilherme Schymura de Oliveira, Renato Fragelli Cardoso, Sérgio Ribeiro da Costa Werlang e Uriel de Magalhães - Maio de 1994 - 106 pág.

239. PUBLIC EXPENDITURES, TAXATION AND WELFARE MEASUREMENT - Pedro Cavalcanti Ferreira - Maio de 1994 - 36 pág.

240. A NOTE ON POLICY, THE COMPOSITION OF PUBLIC EXPENDITURES AND ECONOMIC GROWTH - Pedro Cavalcanti Ferreira - Maio de 1994 - 40 pág. (esgotado)

241. INFLAÇÃO E O PLANO FHC - Rubens Penha Cysne - Maio de 1994 - 26 pág. (esgotado)

242. INFLATIONARY BIAS AND STATE OWNED FINANCIAL INSTITUTIONS - Walter Novaes Filho e Sérgio Ribeiro da Costa Werlang - Junho de 1994 -35 pág.

243. INTRODUÇÃO À INTEGRAÇÃO ESTOCÁSTICA - Paulo Klinger Monteiro - Junho de 1994 - 38 pág. (esgotado)

244. PURE ECONOMIC THEORIES: THE TEMPORARY HALF-TRUTH - Antonio M. Silveira - Junho de 1994 - 23 pág. (esgotado)

245. WELFARE COSTS OF INFLATION - THE CASE FOR INTEREST-BEARING MONEY AND EMPIRICAL ESTIMATES FOR BRAZIL - Mario Henrique Simonsen e Rubens Penha Cysne - Julho de 1994 - 25 pág. (esgotado)

246. INFRAESTRUTURA PÚBLICA, PRODUTIVIDADE E CRESCIMENTO - Pedro Cavalcanti Ferreira - Setembro de 1994 - 25 pág.

247. MACROECONOMIC POLICY AND CREDIBILITY: A COMPARATIVE STUDY OF THE FACTORS AFFECTING BRAZILIAN AND ITALIAN INFLATION AFTER 1970 Giuseppe Tullio e Marcio Ronci - Outubro de 1994 - 61 pág. (esgotado)

248. INFLATION AND DEBT INDEXATION: THE EQUIVALENCE OF TWO ALTERNATIVE SCHEMES FOR THE CASE OF PERIODIC PAYMENTS - Clovis de Faro - Outubro de 1994 -18 pág. 
249. CUSTOS DE BEM ESTAR DA INFLAÇÃO - O CASO COM MOEDA INDEXADA E ESTIMATIVAS EMPÍRICAS PARA O BRASIL - Mario Henrique Simonsen e Rubens Penha Cysne - Novembro de 1994 - 28 pág. (esgotado)

250. THE ECONOMIST MACHIAVELLI - Brena P. M. Fernandez e Antonio M. Silveira Novembro de 1994 - 15 pág.

251. INFRAESTRUTURA NO BRASIL: ALGUNS FATOS ESTILIZADOS - Pedro Cavalcanti Ferreira - Dezembro de 1994 - 33 pág. (esgotado)

252. ENTREPRENEURIAL RISK AND LABOUR'S SHARE IN OUTPUT - Renato Fragelli Cardoso - Janeiro de 1995 - 22 pág.

253. TRADE OR INVESTMENT ? LOCATION DECISIONS UNDER REGIONAL INTEGRATION - Marco Antonio F.de H. Cavalcanti e Renato G. Flôres Jr. - Janeiro de 1995 - 35 pág.

254. O SISTEMA FINANCEIRO OFICIAL E A QUEDA DAS TRANFERÊNCIAS INFLACIONÁRIAS - Rubens Penha Cysne - Janeiro de 1995 - 32 pág. (esgotado)

255. CONVERGÊNCIA ENTRE A RENDA PER-CAPITA DOS ESTADOS BRASILEIROS Roberto G. Ellery Jr. e Pedro Cavalcanti G. Ferreira - Janeiro 1995 - 42 pág.

256. A COMMENT ON "RATIONAL LEARNING LEAD TO NASH EQUILIBRIUM" BY PROFESSORS EHUD KALAI EHUD EHUR - Alvaro Sandroni e Sergio Ribeiro da Costa Werlang - Fevereiro de 1995 - 10 pág.

257. COMMON CYCLES IN MACROECONOMIC AGGREGATES (revised version) - João Victor Issler e Farshid Vahid - Fevereiro de 1995 - 57 pág.

258. GROWTH, INCREASING RETURNS, AND PUBLIC INFRASTRUCTURE: TIMES SERIES EVIDENCE (revised version) - Pedro Cavalcanti Ferreira e João Victor Issler Março de 1995 - 39 pág.(esgotado)

259. POLÍTICA CAMBIAL E O SALDO EM CONTA CORRENTE DO BALANÇO DE PAGAMENTOS - Anais do Seminário realizado na Fundação Getulio Vargas no dia 08 de dezembro de 1994 - Rubens Penha Cysne (editor) - Março de 1995 - 47 pág. (esgotado)

260. ASPECTOS MACROECONÔMICOS DA ENTRADA DE CAPITAIS - Anais do Seminário realizado na Fundação Getulio Vargas no dia 08 de dezembro de 1994 - Rubens Penha Cysne (editor) - Março de 1995 - 48 pág. (esgotado)

261. DIFICULDADES DO SISTEMA BANCÁRIO COM AS RESTRIÇÕES ATUAIS E COMPULSÓRIOS ELEVADOS - Anais do Seminário realizado na Fundação Getulio Vargas no dia 09 de dezembro de 1994 - Rubens Penha Cysne (editor) - Março de 1995 47 pág. (esgotado)

262. POLÍTICA MONETÁRIA: A TRANSIÇÃO DO MODELO ATUAL PARA O MODELO CLÁSSICO - Anais do Seminário realizado na Fundação Getulio Vargas no dia 09 de dezembro de 1994 - Rubens Penha Cysne (editor) - Março de 1995 - 54 pág. (esgotado)

263. CITY SIZES AND INDUSTRY CONCENTRATION - Afonso Arinos de Mello Franco Neto - Maio de 1995 - 38 pág.

264. WELFARE AND FISCAL POLICY WITH PUBLIC GOODS AND INFRASTRUCTURE (Revised Version) - Pedro Cavalcanti Ferreira - Maio de 1995 - 33 pág. 
265. PROFIT SHARING WITH HETEROGENEOUS ENTREPRENEURIAL PROWESS Renato Fragelli Cardoso - Julho de 1995 - 36 pág.

266. A DINÂMICA MONETÁRIA DA HIPERINFLAÇÃO: CAGAN REVISITADO - Femando de Holanda Barbosa - Agosto de 1995 - 14 pág. (esgotado)

267. A SEDIÇÃO DA ESCOLHA PÚBLICA: VARIAÇÕES SOBRE O TEMA DE REVOLUÇÕES CIENTÍfICAS - Antonio Maria da Silveira - Agosto de 1995 - 24 pág.

268. A PERSPECTIVA DA ESCOLHA PÚBLICA E A TENDÊNCIA INSTITUCIONALISTA DE KNIGHT - Antonio Maria da Silveira - Setembro de 1995 - 28 pág.

269. ON LONG-RUN PRICE COMOVEMENTS BETWEEN PAINTINGS AND PRINTS Renato Flôres - Setembro de 1995 - 29 pág.

270. CRESCIMENTO ECONÔMICO, RENDIMENTOS CRESCENTES E CONCORRÊNCIA MONOPOLISTA - Pedro Cavalcanti Ferreira e Roberto Ellery Junior - Outubro de 1995 - 32 pág. (esgotado)

271. POR UMA CIÊNCIA ECONÔMICA FILOSOFICAMENTE INFORMADA: A INDETERMINAÇÃO DE SENIOR - Antonio Maria da Silveira - Outubro de 1995 - 25 pág. (esgotado)

272. ESTIMATING THE TERM STRUCTURE OF VOLATILITY AND FIXED INCOME DERIVATIVE PRICING - Franklin de O. Gonçalves e João Victor Issler - Outubro de 1995 - 23 pág. (esgotado)

273. A MODEL TO ESTIMATE THE US TERM STRUCTURE OF INTEREST RATES Antonio Marcos Duarte Júnior e Sérgio Ribeiro da Costa Werlang - Outubro de 1995 - 21 pág. (esgotado)

274. EDUCAÇÃO E INVESTIMENTOS EXTERNOS COMO DETERMINANTES DO CRESCIMENTO A LONGO PRAZO - Gustavo Gonzaga, João Victor Issler e Guilherme Cortella Marone - Novembro de 1995 - 34 pág.

275. DYNAMIC HEDONIC REGRESSIONS: COMPUTATION AND PROPERTIES - Renato Galvão Flôres Junior e Victor Ginsburgh - Janeiro de 1996 - 21 pág.

276. FUNDAMENTOS DA TEORIA DAS OPÇÕES - Carlos Ivan Simonsen Leal - Fevereiro de 1996 - 38 pág. (esgotado)

277. DETERMINAÇÃO DO PREÇO DE UMA OPÇÃO E ARBITRAGEM - Carlos Ivan Simonsen Leal - Fevereiro 1996 - 55 pág. (esgotado)

278. SUSTAINED GROWTH, GOVERNMENT EXPENDITURE AND INFLATION - Pedro Cavalcanti Ferreira - Fevereiro 1996 - 38 pág.

279. REFLEXOS DO PLANO REAL SOBRE O SISTEMA BANCÁRIO BRASILEIRO Rubens Penha Cysne e Sérgio Gustavo Silveira da Costa - Junho 1996 - 28 pág.

280. CURSO DE MATEMÁTICA PARA ECONOMISTAS, CAPÍTULOS I E II: FUNÇÕES, ÁlGEBRA LINEAR E APLICAÇÕES - Rubens Penha Cysne e Humberto de Athayde Moreira - Junho 1996 - 75 pág. (esgotado)

281. PREVIDÊNCIA COMPLEMENTAR PATROCINADA: VALE A PENA? - Clovis de Faro e Moacyr Fioravante - Junho de 1996 - 23 pág. 
282. OLIGOPOLISTIC COMPETITION UNDER KNIGHTIAN UNCERTAINTY - Hugo Pedro Boff e Sérgio Ribeiro da Costa Werlang - Julho de 1996 - 37 pág.

283. CURSO DE MATEMÁTICA PARA ECONOMISTAS - CAPÍTULO IV: OTIMIZAÇÃO ESTÁTICA - Rubens Penha Cysne e Humberto de Athayde Moreira - Julho de 1996 - 71 pág.

284. RIO DE JANEIRO E INTERMEDIAÇÃO FINANCEIRA - Rubens Penha Cysne - Julho de 1996 - 30 pág.

285. CURSO DE MATEMÁTICA PARA ECONOMISTAS CAPÍTULO III: CÁLCULO NO $\mathrm{R}^{\mathrm{n}}$ - Rubens Penha Cysne e Humberto Athayde Moreira - Agosto de 1996 - 106 pág.

286. REFLEXOS DO PLANO REAL SOBRE AS FINANCEIRAS - Rubens Penha Cysne e Sergio Gustavo S. da Costa - Setembro de 1996 - 17 pág. (esgotado)

287. FUTUROS DE JUROS - Carlos Ivan Simonsen Leal - Setembro de 1996 - 49 pág.

288. PREVIDÊNCIA SOCIAL NO BRASIL: POR UMA REFORMA MAIS DURADOURA Clovis de Faro - Setembro de 1996 - 38 pág.

289. CURSO DE MATEMÁTICA PARA ECONOMISTA - CAPÍTULO V: OTIMIZAÇÃO DINÂMICA - Rubens Penha Cysne e Humberto de Athayde Moreira - Setembro de 1996 60 pág.

290. PERSPECTIVAS DE LONGO PRAZO DA ECONOMIA BRASILEIRA: UMA ANÁLISE EXPLORATÓRIA - Pedro C. Ferreira - Outubro de 1996 - 40 pág.

291. INTEGRAÇÃO, CRESCIMENTO E BEM-ESTAR - Marcelo Leite de Moura e Silva e Pedro C. Ferreira - Outubro de 1996 - 39 pág. 


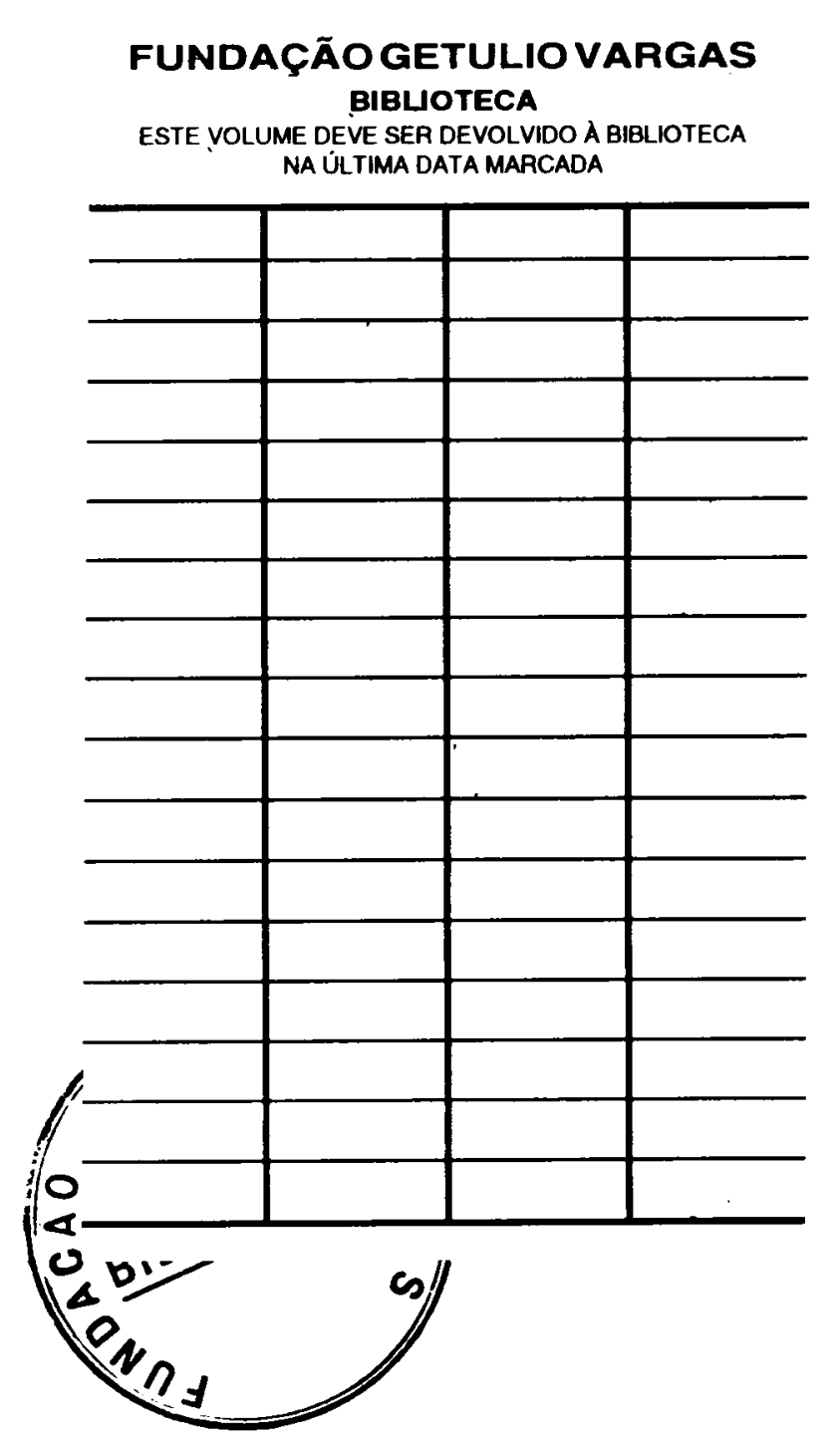

N.Cham. P/EPGE EE 291

Autor: SILVA, Marcelo Leite de Moura e Título: Integração, crescimento e bem-estar.

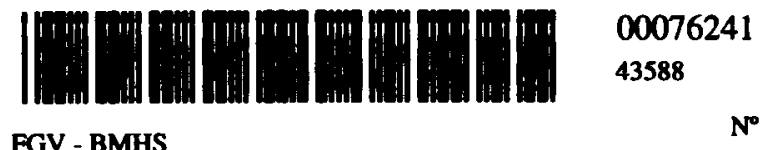

